\title{
Genome-Wide Association Study Identifies Novel Candidate Genes for Aggressiveness, Deoxynivalenol Production, and Azole Sensitivity in Natural Field Populations of Fusarium graminearum
}

\author{
Firas Talas, Rasha Kalih, Thomas Miedaner, and Bruce A. McDonald
}

Submitted 5 October 2015. Accepted 26 February 2016.

\begin{abstract}
Genome-wide association studies can identify novel genomic regions and genes that affect quantitative traits. Fusarium head blight is a destructive disease caused by Fusarium graminearum that exhibits several quantitative traits, including aggressiveness, mycotoxin production, and fungicide resistance. Restriction site-associated DNA sequencing was performed for 220 isolates of $F$. graminearum. A total of 119 isolates were phenotyped for aggressiveness and deoxynivalenol (DON) production under natural field conditions across four environments. The effective concentration of propiconazole that inhibits isolate growth in vitro by $50 \%$ was calculated for 220 strains. Approximately 29,000 single nucleotide polymorphism markers were associated to each trait, resulting in 50, 29, and 74 quantitative trait nucleotides (QTNs) that were significantly associated to aggressiveness, DON production, and propiconazole sensitivity, respectively. Approximately 41\% of these QTNs caused nonsynonymous substitutions in predicted exons, while the remainder were synonymous substitutions or located in intergenic regions. Three QTNs associated with propiconazole sensitivity were significant after Bonferroni correction. These QTNs were located in genes not previously associated with azole sensitivity. The majority of the detected QTNs were located in genes with predicted regulatory functions, suggesting that nucleotide variation in regulatory genes plays a major role in the corresponding quantitative trait variation.
\end{abstract}

Genome-wide association studies (GWAS) provide a powerful tool to determine the genetic basis of quantitative traits. GWAS are commonly utilized in model eucaryotes such as humans (Frazer et al. 2009; McCarthy et al. 2008; Rietveld et al. 2013), Drosophila (Burke et al. 2014), and yeast (Nelson et al. 2013), but has rarely been applied to filamentous fungi. In Neurospora crassa, in vitro microbial communication among 24 strains was measured using confocal microscopy and was coupled with 81,614 single nucleotide polymorphisms (SNPs) to identify a gene significantly associated with the trait (PalmaGuerrero et al. 2013). A GWAS analysis utilizing 23 strains of the spruce and pine tree pathogen Heterobasidion annosum

Corresponding author: F. Talas: E-mail: firas.talas@usys.ethz.ch

*The $\boldsymbol{e}$-Xtra logo stands for "electronic extra" and indicates that one supplementary table is published online.

(C) 2016 The American Phytopathological Society identified 12 candidate virulence genes based on 33,018 SNP markers (Dalman et al. 2013).

A major limitation in all studies oriented around quantitative traits is the ability to make precise and reproducible measures of the relevant phenotype. To improve trait reproducibility, most studies measure phenotypes in highly controlled laboratory or greenhouse environments and focus on a small number of experimental strains. Because the natural field environment is prone to fluctuations that are likely to affect a quantitative trait and field populations are often composed of thousands of genetically differentiated pathogen strains, it is not surprising that candidate genes identified in laboratory studies may not show the expected effects when studied in natural field populations (Barrett and Hoekstra 2011; Xiao et al. 2009). A different approach to identify the genes underlying the traits that are most relevant in agriculture is to focus on natural, standing genetic variation in natural pathogen populations that have already been selected under field conditions, as opposed to variation that was generated through mutagenesis or selected under artificial laboratory conditions. A second way to increase the likely functional relevance of genes identified through GWAS is to generate the phenotype data using replicated trials conducted in the natural field environment.

Fusarium graminearum [teleomorph Gibberella zeae (Schweinitz) Petch] is a haploid, ascomycete fungus causing Fusarium head blight (FHB). The genome sequence revealed four chromosomes encompassing approximately $36 \mathrm{Mbp}$ and 14,000 identified gene models (Cuomo et al. 2007; King et al. 2015). As for most fungi, the majority of gene models have no known function. FHB is an important disease on cereals globally, causing both significant yield losses and mycotoxin contamination. Fungicides can provide up to $70 \%$ reductions in disease when applied at or shortly after infection (Wagacha and Muthomi 2007), but fungicide resistance is likely to emerge following regular exposure (Dubos et al. 2011; Talas and McDonald 2015a). The largest group of fungicides used to control FHB is the demethylation inhibitors (DMI) that inhibit a $14 \alpha$-sterol demethylase needed to synthesize ergosterol, the primary sterol in fungal cell membranes. Point mutations in the CYP51 gene were associated with DMI resistance in the wheat pathogen Zymoseptoria tritici (Brunner et al. 2008), but we could not find an association between CYP51 sequence variation and DMI sensitivity in an earlier study (Talas and McDonald 2015a), suggesting that other genes are likely responsible for the differences in DMI sensitivity observed in the 224 field strains of $F$. graminearum included in this analysis.

Aggressiveness in $F$. graminearum was associated with two mitogen-activated protein kinase (MAPK) genes, $M G V 1$ and GPMK1 (Hou et al. 2002), and one lipase-encoding gene, FGL1 
(Voigt et al. 2005). Other kinase genes were also reported to affect $F$. graminearum aggressiveness (Qin et al. 2015). FHB infection is usually associated with mycotoxin contamination and a close relationship was found between trichothecene production and aggressiveness (Becher et al. 2013). Although trichothecene was not essential to initiate the infection, it appears to enhance spread within colonized tissues (Boenisch and Schäfer 2011). Different genes involved in trichothecene biosynthesis are mainly clustered in a 26-kbp region called the TRI5 cluster (McCormick et al. 2004). RAS (GTPase-encoding) genes regulate MAPK genes and are considered essential for aggressiveness in F. graminearum. Disruption of RAS2 led to slower growth on media, delayed spore germination, and a significant reduction of aggressiveness in wheat and maize (Bluhm et al. 2007).

An earlier attempt to associate nucleotide polymorphism with variation in aggressiveness and deoxynivalenol (DON) production used a candidate gene association approach. This study revealed two quantitative trait nucleotides (QTNs) within genes MetAPI (FGSG_08531) and Erf2 (FGSG_01397), explaining 25.6 and $13.1 \%$ of the aggressiveness variation respectively, and one QTN within gene Tril (FGSG_00071), explaining 4.4\% of the variance in DON production (Talas et al. 2012b). Tril encodes a cytochrome P450 monooxygenase that contributes to trichothecene biosynthesis along with the 12 biosynthetic genes located in the TRI5 cluster (Meek et al. 2003), while Erf2 is thought to regulate subcellular localization of the RAS protein (Bartels et al. 1999; Bluhm et al. 2007). While candidate gene association can detect the quantitative effects of particular SNPs within a restricted number of previously identified genes, it cannot identify novel genes affecting a trait. A forwardgenetics approach such as GWAS or QTL mapping is needed to identify novel genes.

Restriction site-associated DNA sequencing (RADseq) can provide large numbers of SNP genetic markers distributed across a genome at a relatively low cost (Baird et al. 2008). Our objective in this study was to use GWAS to identify genomic regions and candidate genes associated with aggressiveness, DON production, and propiconazole resistance in a natural population of $F$. graminearum. An important aspect of our approach was to use phenotype data collected in four distinct field environments from 119 wild-type strains sampled from a large geographical region, allowing us to identify genomic regions and candidate genes that are likely to play a significant role in aggressiveness and DON production in agroecosystems.

\section{RESULTS}

Phenotypes and genetic markers fit to the GWAS model.

The phenotypes aggressiveness, DON production, and propiconazole sensitivity followed a normal distribution (Fig. 1) with mean values of $29 \%, 14.8 \mathrm{mg} \mathrm{kg}^{-1}$, and $22.2 \mathrm{mg} \mathrm{l}^{-1}$, with heritabilities $\left(H^{2}\right)$ of $0.61,0.67$, and 0.97 , respectively. The variance components analyses (Table 1) revealed significant variation among different environments, isolates, and their interaction (isolates $\times$ environment). Variance among different isolates was significantly higher than variance among different replicates of the same isolate. The variance component associated with replicates (i.e., within each environment or biological repeat) did not show significant variation for any of the traits, indicating an absence of cross infection from neighboring plots or cross-contamination among wells in microtiter plates. Aggressiveness and DON production are affected by temperature and humidity; hence, it was not surprising to find significant variation among different environments in our field experiment (Table 1).

Quality filtering resulted in 36,283 high-quality SNPs shared among 220 isolates included in the analyses. The SNP densities were equivalent across the four chromosomes and proportional to their relative sizes. Additional filters for minimum and maximum allele frequencies, maximum missing data, and linkage reduced the number to approximately 29,000 SNPs analyzed for each trait. Based on principal coordinates analysis, no population structure was detected among the isolates used in

Table 1. Analysis of variance to differentiate components contributing to phenotypic variation in aggressiveness and deoxynivalenol (DON) production

\begin{tabular}{lrrrrrr}
\hline & & \multicolumn{2}{c}{ Aggressiveness } & & \multicolumn{2}{c}{ DON production } \\
Source of variation & DF & Estimate & Pr $>$ F & & Estimate & Pr $>$ F \\
\hline Environment (Env) & 7 & 18.69 & $<0.0001$ & & 1.62 & $<0.0001$ \\
Replication (Rep) & 1 & 0.99 & 0.0064 & & 0.09 & 0.0916 \\
Isolates (Iso) & 117 & 11.26 & $<0.0001$ & & 0.28 & $<0.0001$ \\
Env $\times$ Iso & 351 & 12.36 & $<0.0001$ & & 0.09 & 0.1661 \\
Error & 470 & 32.98 & & & 0.90 & \\
$H^{2}$ & & 0.61 & & 0.67 & \\
\hline
\end{tabular}

a The data for this trait were BOX-COX transformed.

$\mathrm{b}$ The isolates were nested according to the associated field experiment (2009/2010 and 2011/2012).
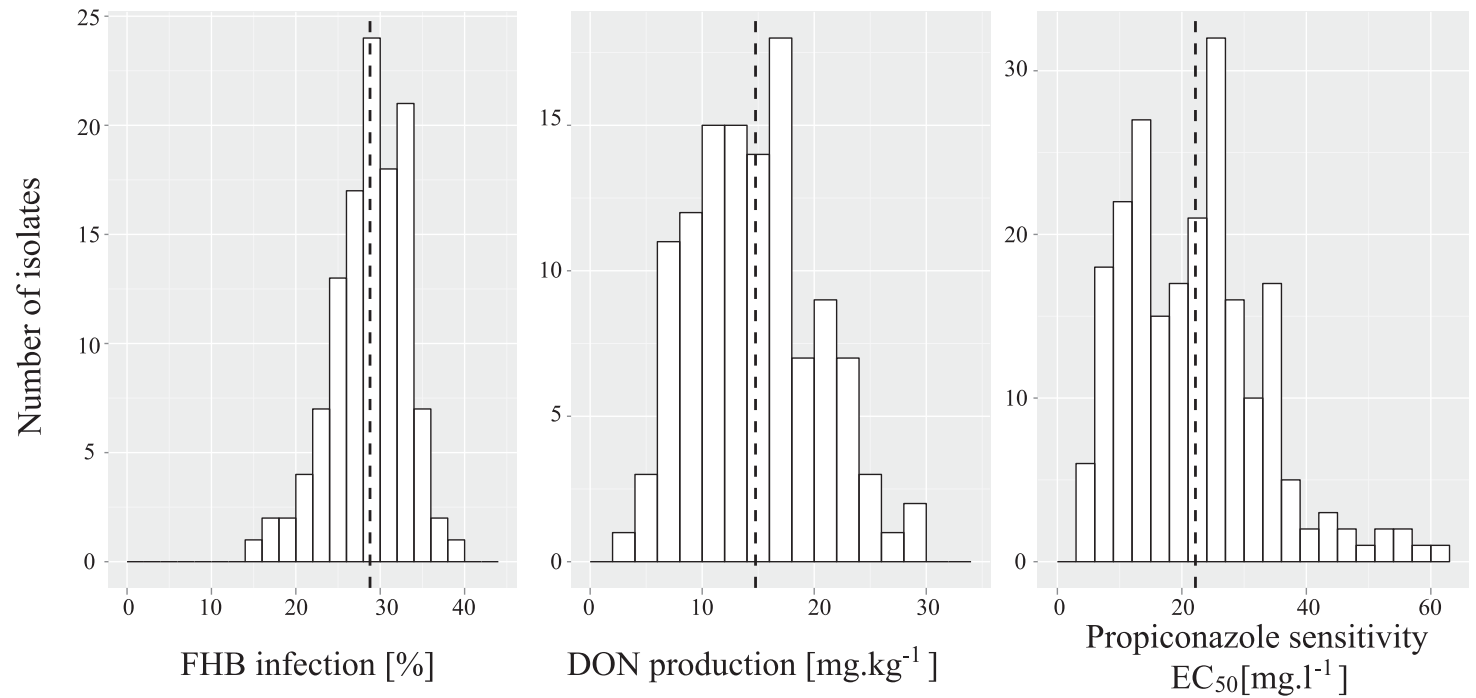

Fig. 1. The distribution of aggressiveness, deoxynivalenol (DON) production, and propiconazole sensitivity phenotypes among the Fusarium graminearum strains included in the genome-wide association studies. The dashed lines represent the mean value of each trait. 
our GWAS population (Talas and McDonald 2015b), although it carried high genetic variation, so we did not need to correct the GWAS model for population structure. However, we corrected our GWAS model for family structure to minimize the rate of false-positive detection. In a preliminary study (Talas and McDonald 2015b), a rapid decay in linkage disequilibrium (LD) was detected over only 1,000 bp, indicating that a GWAS approach would provide high resolution in this population. Due to the nature of the field experiments used for phenotyping, different numbers of isolates were tested for different traits. The final number of isolates analyzed for aggressiveness and DON production under field conditions was 119 , while 220 isolates were analyzed for propiconazole sensitivity in vitro. Therefore, two SNP datasets were generated independently for these two subcollections.

SNPs associated with aggressiveness, DON production, and propiconazole sensitivity.

The mixed linear model analysis generated a significance value for each SNP, using an $F$ test for the null hypothesis that a
SNP has no effect on the measured trait (Zhang et al. 2010) Plots of the negative logarithmic estimates of the observed $P$ values against the expected values (quantile-quantile plots) showed that the model with a kinship correction was a good fit to our datasets (Fig. 2). The correlation between the minimum allele frequency and the logarithmic $P$ value indicated that the QTNs significantly associated with the traits showed a balanced allele frequency among the tested populations (Fig. 3). After applying a mixed linear model with a correction incorporating a kinship matrix within the model to reduce random genetic effects (Kang et al. 2008), we found 1,326 QTNs significantly associated $(P \leq 0.05)$ with aggressiveness, 1,415 with DON production, and 1,298 with propiconazole sensitivity. The significance threshold was then adjusted by setting the false discovery rate (FDR) to a cutoff of 0.05 (Benjamini and Hochberg 1995). This reduced the number of significantly associated SNPs to 50 for aggressiveness, 29 for DON production, and 74 for propiconazole sensitivity (Fig. 4). The significance of three QTNs related to propiconazole sensitivity exceeded the Bonferroni threshold (Hochberg 1988).
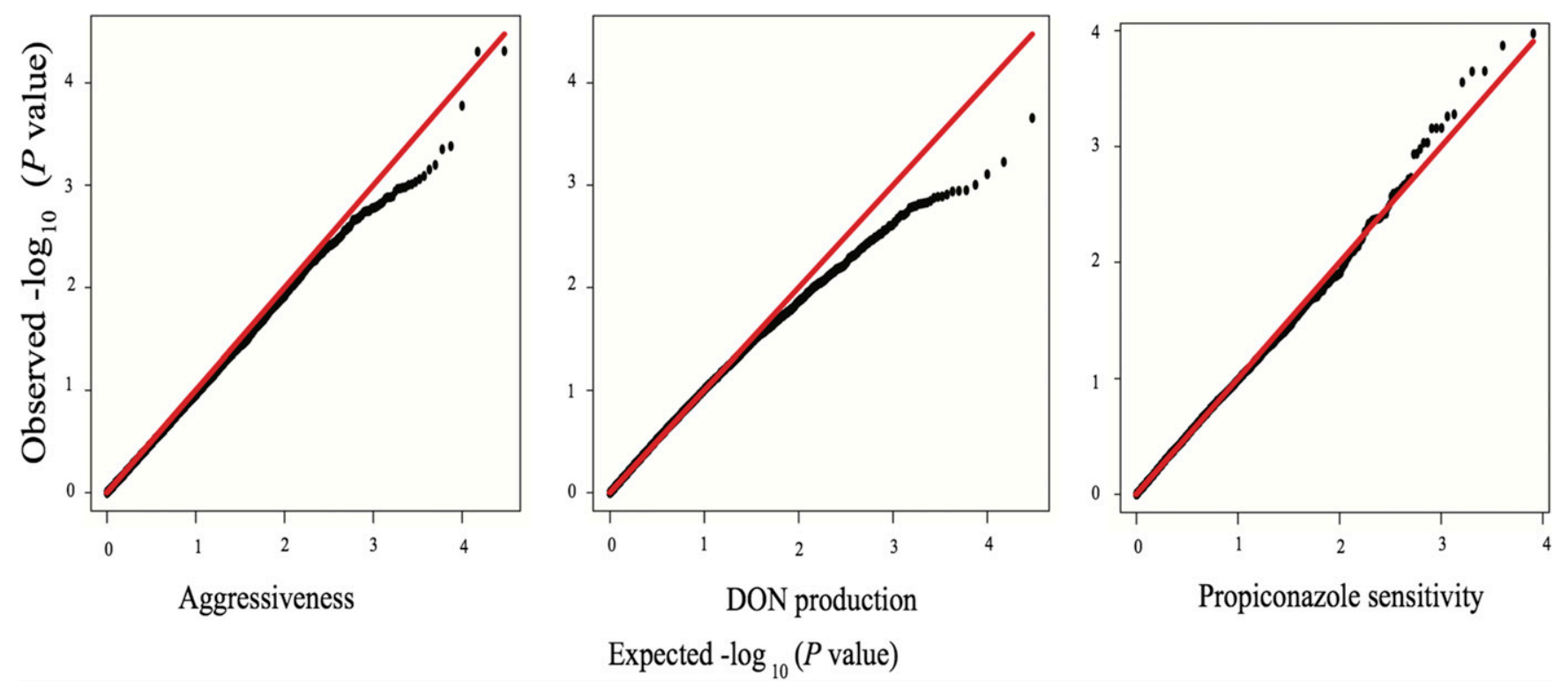

Fig. 2. Quantile-quantile plots between expected and observed $P$ values $(-\log 10)$ for the traits aggressiveness, deoxynivalenol (DON) production, and propiconazole sensitivity (black dots). The straight diagonal lines represent the reference correlation.
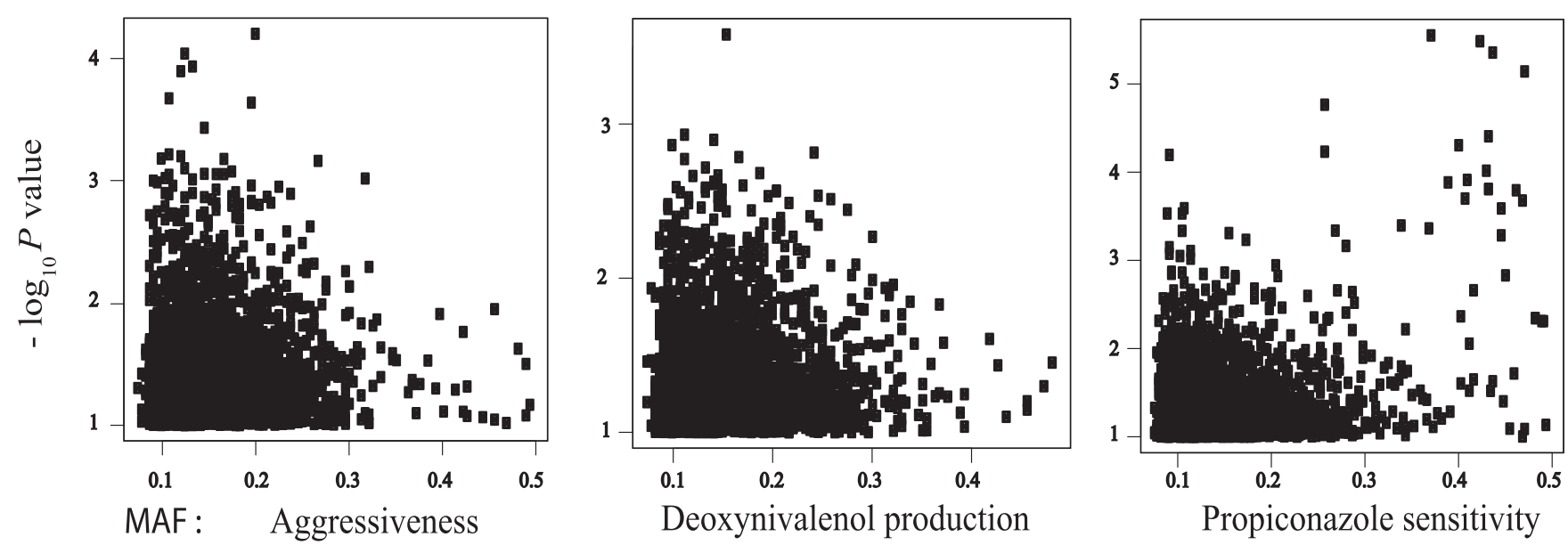

Fig. 3. Relationships between the identified quantitative trait nucleotides for aggressiveness, deoxynivalenol production, and propiconazole sensitivity and the allele frequencies of the corresponding single nucleotide polymorphisms in the tested Fusarium graminearum population. MAF refers to the minimum allele frequency. 
Based on the position of each SNP in the reference genome, we identified the associated genes and the position of the QTN in each gene (Tables 2, 3, 4). Most of the detected QTNs were nonsynonymous substitutions, but there were 7, 3, and 21 synonymous QTNs for aggressiveness, DON production, and propiconazole sensitivity, respectively, while 24,12 , and 23 QTNs, respectively, were located in intergenic regions. Gene annotations were based on domain homologies with other fungi using the PEDANT 3 database (Frishman et al. 2003; Riley et al. 2007). Our results were based on the annotations given in the Munich Information Center for Protein Sequences (MIPS) v.3.2; however, for some QTNs, we were able to identify an annotated gene in the reference genome sequence maintained by the Broad Institute (Cuomo et al. 2007) but not in the MIPS version of the reference genome (Wong et al. 2011). Similar inconsistencies between the Broad and MIPS annotations were reported based on RNAseq analyses of the $\mathrm{PH}-1$ reference isolate (Zhao et al. 2013). A recently published update on the genome sequence of $F$. graminearum highlighted these differences (King et al. 2015).

Aggressiveness was associated with 19 nonsynonymous substitutions located in 19 genes, while seven other genes contained synonymous substitutions. The most significant QTN related to aggressiveness was located in a gene (FGSG_00355) predicted to encode an activating protein of RAS-GTPase. Another aggressiveness-associated gene (FGSG_08010) was reported to be up-regulated during $F$. graminearum infection (Lawler et al. 2013). However, the other genes associated with aggressiveness were not identified in earlier studies of the infection process of $F$. graminearum, though domains predicted for the corresponding proteins often have a regulatory function (e.g., FGSG_00586, FGSG_13008, FGSG_01339, FGSG_02531, FGSG_05475).

Among the 17 genes associated with DON production, only FGSG_01943 was previously reported to be up-regulated during F. graminearum infection (Stephens et al. 2008). The remaining genes were not previously characterized in $F$. graminearum, except for their predicted function based on domain homology.

Three of the 51 genes significantly associated with propiconazole sensitivity in the GWAS exceeded the Bonferroni significance threshold (FGSG_06044, FGSG_08585, FGSG_06938). Eight genes contained domains expected to regulate transcription (FGSG_06044, FGSG_06938, FGSG_09919, FGSG_11696, FGSG_08397, FGSG_00627, FGSG_02251, FGSG_05884). One gene was related to a polyketide synthase (FGSG_04588) and six genes contained domains predicted to have a transport function (FGSG_02619, FGSG_01612, FGSG_12042, FGSG_06453, FGSG_01166, FGSG_03957). One gene encoded an ABC
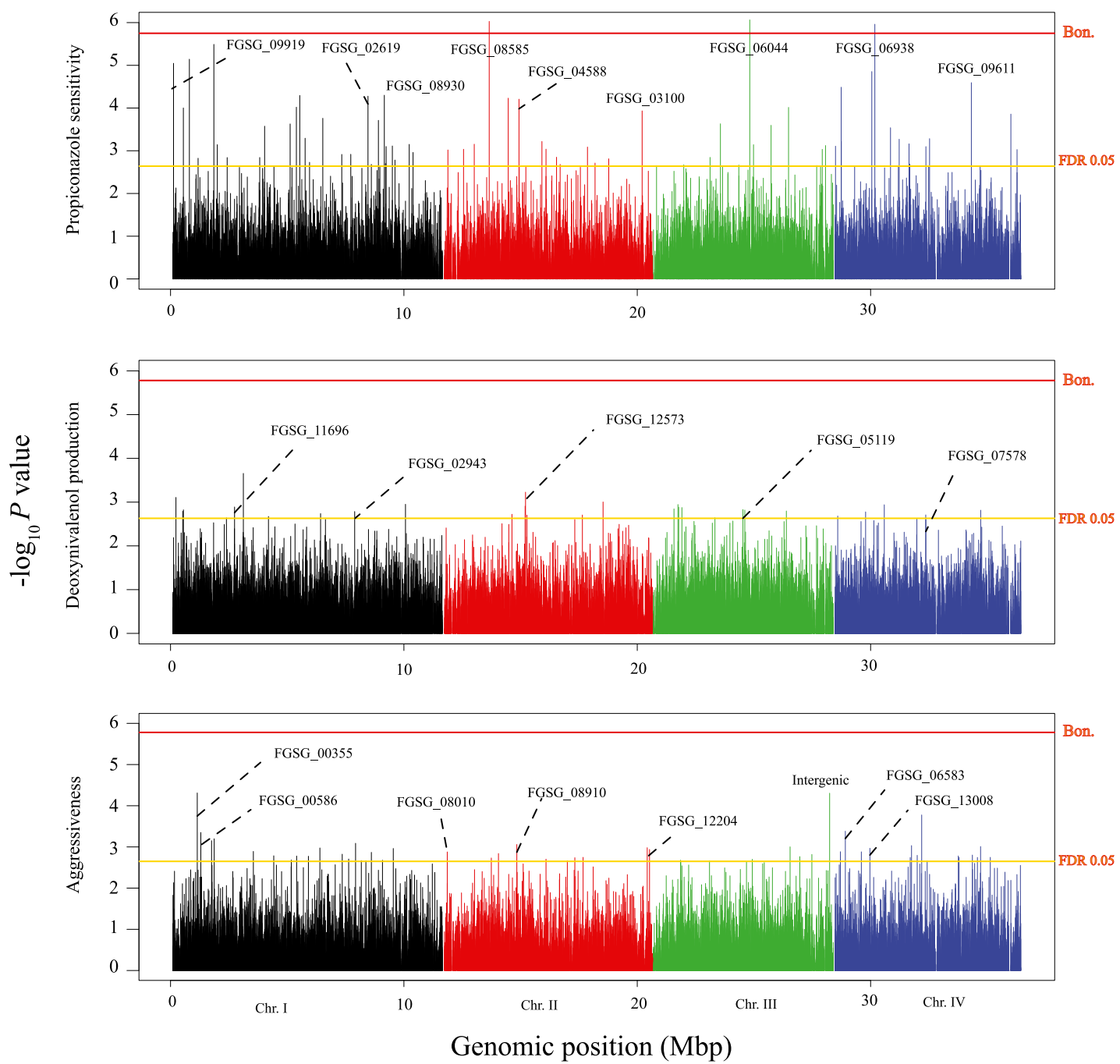

Fig. 4. The positions of the analyzed RADseq single nucleotide polymorphisms across the PH-1 Fusarium graminearum reference genome sequence and their respective associations with aggressiveness, deoxynivalenol production, and propiconazole sensitivity. The upper solid line in each graph shows the Bonferroni threshold and the lower line shows the false discovery rate (FDR) threshold of 0.05 . 
transporter cassette (FGSG_09611). Three genes (FGSG_06453, FGSG_03330, FGSG_03100) contained domains of a major facilitator super family (MFS). The protein homologs of FGSG_06453 and FGSG_03330 were related to fungicide resistance in other pathogenic fungi, according to MIPS v.3.2 (Wong et al. 2011).

\section{DISCUSSION}

Many uncharacterized genes in the published draft of the F. graminearum genome sequence are likely to affect important agronomic traits such as aggressiveness, mycotoxin production, thermal adaptation, and fungicide resistance. Based on gene homology, Wang et al. (2011) used gene knockouts in the genome reference strain $\mathrm{PH}-1$, combined with virulence tests in a greenhouse, to identify 31 protein kinase genes that affected pathogenicity. We did not identify any of these genes in our analyses. A well-structured GWAS provides a forward-genetics approach to identify novel candidate genes and regulatory sequences worthy of further molecular and biological characterization. The statistical associations between SNP markers and traits identified in this study will require additional studies oriented around functional validation in order to draw a causative conclusion. While greenhouse and growth-chamber experiments conducted in a uniform environment offer many experimental advantages, the resulting phenotypes may not accurately reflect the interactions occurring between a pathogen strain and its host under natural field conditions characterized by a continuously changing environment (e.g., with continuous fluctuations in temperature, humidity, radiation, and rainfall). Because our quantitative phenotypes were measured across many wild-type strains carrying natural genetic variation under natural field conditions in field experiments representing four different field environments, we believe that our GWAS analyses are more likely to identify chromosomal segments and corresponding genes that will be relevant in farmers' fields under real-world conditions.

\section{Measures of trait variation.}

Significant genetic variance $(P<0.0001)$ was found among isolates for aggressiveness and DON production under natural field conditions. The variance associated with different environments was significant, including differences among years, locations, and position of the experiment within the experimental field sites (Table 1). This variance likely reflects different microenvironments associated with each experimental context. Analysis of variance was used to separate the different variance components and enabled us to calculate the broad-sense heritability associated with each trait. A surprisingly high heritability was found under natural field conditions for aggressiveness $\left(H^{2}=0.61\right)$ and DON production $\left(H^{2}=\right.$ $0.67)$. The high heritability $\left(H^{2}=0.97\right)$ associated with propiconazole sensitivity measured under tightly controlled laboratory conditions was reported earlier (Talas and McDonald 2015a).

Our GWAS analysis identified a large number of QTNs associated with each trait $(110,74$, and 168 QTNs for aggressiveness, DON production, and propiconazole sensitivity, respectively) by applying the traditional FDR with a 0.10 cutoff; hence, we raised the significance threshold by applying FDR with a 0.05 cutoff, resulting in loss of around half of the QTNs for each trait. Several of the remaining significant QTNs were located in intergenic regions or could not be assigned to an annotated gene (i.e., 24, 12, and 23 QTNs for aggressiveness, DON production, and propiconazole sensitivity, respectively). These QTNs may represent regulatory elements or mis-annotated genes (Zhao et al. 2013). They may also represent SNPs that are in LD with nearby genes or regulatory sequences that are responsible for the trait variance. Similarly, QTNs encoding synonymous substitutions may be in LD with other regions of the same gene or neighboring genes that carry nonsynonymous substitutions responsible for the observed trait variance.

\section{QTNs associated with aggressiveness.}

GWAS analysis identified a highly significant QTN explaining $24 \%$ of the measured variance in aggressiveness within the gene FGSG_00355 on chromosome 1 (Fig. 4; Table 2). This gene encodes a RAS-GTPase activating protein. The GTPase activating proteins Ira1 and Ira2 in Saccharomyces cerevisiae negatively regulate $R A S 1$ and RAS2 (Harashima et al. 2006), which play important roles in yeast longevity (Sun et al. 1994). RAS2 influences a MAPK cascade and cell morphology in addition to fungal pathogenicity in Ustilago maydis (Lee and Kronstad 2002). Similarly, RAS2 affects growth and aggressiveness in $F$. graminearum by regulating the Gpmkl MAPK pathway and plays a minor role in cAMP signaling (Bluhm et al. 2007). In an earlier candidate gene association study, FGSG_08531 (Erf2), another gene associated with the RAS protein, explained $13 \%$ of the aggressiveness found in a subset of these $F$. graminearum isolates (Talas et al. 2012b). Hence, we associated aggressiveness with natural variation in two genes regulating the RAS protein in two different studies involving the same group of isolates. This indicates that $F$. graminearum aggressiveness in the field is significantly affected by the RAS protein, likely through its effects on the associated MAPK pathway.

Gene FGSG_08910 explained approximately $18 \%$ of the genetic variance in aggressiveness. This gene contains a CAS/CSE domain (Finn et al. 2014). CAS is involved in cellular apoptosis (programed cell death [PCD]) and proliferation (Brinkmann et al. 1996). It was reported that proper regulation of PCD is important for pathogenicity in Botrytis cinerea (Shlezinger et al. 2012). However, Brinkmann et al. (1996) speculated that CAS may have a regulatory function in the MAPK system, based on its intracellular colocalization on microtubules and the mitotic spindle together with the CAS putative recognition site for MAPK.

QTNs located within genes FGSG_00586, FGSG_13008, FGSG_02531, and FGSG_06075 explained 18, 15, 12, and $15 \%$ of the genetic variance, respectively. All of these genes are predicted to encode zinc binding. The first three genes contain a domain associated with zinc-finger transcription factors. However, this gene family is associated with many biological functions, including amino acid metabolism, mitosis, and meiosis. Superoxide dismutases are zinc-binding proteins that were associated with virulence in $C$. albicans (Frohner et al. 2009). Analyses of transcriptional networks in $F$. graminearum identified regulators associated with coexpression of certain genes (Lawler et al. 2013). Based on gene expression measured in microarrays, FGSG_08010 (associated with $13 \%$ of the genetic variance in aggressiveness) was shown to regulate the expression of two different groups of genes that were up-regulated during infection of a susceptible host (Lawler et al. 2013). This gene encodes a zinc finger-binding domain unique to fungi that regulates genes affecting metabolism and development. Our GWAS based on natural, standing genetic variation found in field populations supports the findings of Lawler et al. (2013). Given the relatively large number of candidate genes associated with zinc binding, we speculate that zinc plays an important role in aggressiveness of $F$. graminearum.

Overall, most of the genes associated with aggressiveness in the GWAS are involved in regulation or transport activities (Table 2). For example, FGSG_01339 encodes a TATA binding protein that is also likely to affect gene regulation. Similarly, 
FGSG_05475 and FGSG_08608 are predicted to have regulatory functions.

\section{QTNs associated with DON production.}

Our GWAS identified 17 genes significantly associated with DON production. Four of these genes encoded uncharacterized hypothetical proteins in $F$. graminearum, though these genes encode domains that may provide insight into their function. Interestingly, the most significant QTN (explaining 19\% of the genetic variance) was assigned to an intergenic region on chromosome 1 , consistent with a regulatory function, a misannotation, or LD with an explanatory polymorphism. A QTN explaining $12 \%$

Table 2. Significant quantitative trait nucleotide polymorphisms in exons associated (at a false discovery rate threshold of 0.05 ) with aggressiveness in Fusarium graminearum

\begin{tabular}{|c|c|c|c|c|c|c|c|c|}
\hline Chra $^{a}$ & SNPa $^{a}$ & $\begin{array}{l}\text { Alternative } \\
\text { allele }\end{array}$ & $\begin{array}{c}\text { Reference } \\
\text { alleles }\end{array}$ & $\begin{array}{l}\text { Position } \\
\text { in gene }\end{array}$ & Substitution & Frequency & $P$ value & $\begin{array}{c}\text { Explained } \\
\text { genetic variance }\end{array}$ \\
\hline 4 & SNP24237 & $\mathrm{T}$ & $\mathrm{C}$ & 3518 & $\mathrm{~A} / \mathrm{V}$ & 0.19 & 0.000415 & 0.25 \\
\hline 1 & SNP00852 & $\mathrm{C}$ & $\mathrm{T}$ & 1781 & $\mathrm{M} / \mathrm{T}$ & 0.11 & 0.000049 & 0.24 \\
\hline 1 & SNP01623 & $\mathrm{T}$ & A & 18 & Syn (R) & 0.19 & 0.000634 & 0.18 \\
\hline 1 & SNP05838 & $\mathrm{A}$ & $\mathrm{T}$ & 2323 & $\mathrm{~S} / \mathrm{T}$ & 0.22 & 0.001058 & 0.18 \\
\hline 2 & SNP12505 & A & $\mathrm{T}$ & 574 & $\mathrm{~S} / \mathrm{T}$ & 0.18 & 0.000873 & 0.18 \\
\hline 2 & SNP14236 & $\mathrm{G}$ & $\mathrm{C}$ & 641 & $\mathrm{~T} / \mathrm{R}$ & 0.08 & 0.002168 & 0.18 \\
\hline 4 & SNP25273 & $\mathrm{C}$ & $\mathrm{G}$ & 1300 & $\mathrm{~A} / \mathrm{P}$ & 0.14 & 0.001076 & 0.18 \\
\hline 1 & SNP07026 & A & $\mathrm{T}$ & 593 & $\mathrm{~F} / \mathrm{Y}$ & 0.16 & 0.000815 & 0.17 \\
\hline 2 & SNP16744 & $\mathrm{G}$ & $\mathrm{T}$ & 557 & $\mathrm{~V} / \mathrm{G}$ & 0.09 & 0.001043 & 0.17 \\
\hline 3 & SNP17675 & $\mathrm{G}$ & $\mathrm{A}$ & 1400 & $\mathrm{H} / \mathrm{R}$ & 0.20 & 0.002099 & 0.17 \\
\hline 2 & SNP14402 & $\mathrm{C}$ & $\mathrm{T}$ & 2058 & Syn (D) & 0.16 & 0.001825 & 0.16 \\
\hline 3 & SNP23376 & A & G & 775 & $\mathrm{~A} / \mathrm{T}$ & 0.11 & 0.001521 & 0.16 \\
\hline 1 & SNP04014 & $\mathrm{G}$ & $\mathrm{A}$ & 1552 & $\mathrm{I} / \mathrm{V}$ & 0.14 & 0.001638 & 0.15 \\
\hline 3 & SNP23037 & G & A & 5447 & $\mathrm{D} / \mathrm{G}$ & 0.11 & 0.001716 & 0.15 \\
\hline 3 & SNP20829 & $\mathrm{T}$ & $\mathrm{C}$ & 2549 & $\mathrm{~A} / \mathrm{V}$ & 0.08 & 0.001996 & 0.15 \\
\hline 3 & SNP18861 & $\mathrm{T}$ & G & 1116 & $\mathrm{Q} / \mathrm{H}$ & 0.10 & 0.002183 & 0.15 \\
\hline 4 & SNP29421 & $\mathrm{G}$ & $\mathrm{A}$ & 79 & $\mathrm{I} / \mathrm{V}$ & 0.16 & 0.001796 & 0.15 \\
\hline 1 & SNP06634 & A & $\mathrm{G}$ & 515 & G/D & 0.12 & 0.0015 & 0.14 \\
\hline 1 & SNP06829 & A & $\mathrm{T}$ & 956 & $\mathrm{~V} / \mathrm{D}$ & 0.16 & 0.001962 & 0.14 \\
\hline 1 & SNP04746 & G & A & 2961 & Syn (R) & 0.15 & 0.002083 & 0.14 \\
\hline 2 & SNP11506 & G & $\mathrm{C}$ & 2445 & Syn (G) & 0.16 & 0.001854 & 0.14 \\
\hline 2 & SNP10101 & $\mathrm{C}$ & $\mathrm{A}$ & 1308 & $\mathrm{Q} / \mathrm{H}$ & 0.09 & 0.001321 & 0.13 \\
\hline 2 & SNP14633 & $\mathrm{C}$ & $\mathrm{T}$ & 1239 & Syn (N) & 0.23 & 0.001787 & 0.13 \\
\hline 1 & SNP07224 & G & A & 348 & Syn (E) & 0.16 & 0.00215 & 0.12 \\
\hline 4 & SNP29198 & A & $\mathrm{G}$ & 41 & $\mathrm{R} / \mathrm{H}$ & 0.26 & 0.001574 & 0.09 \\
\hline 4 & SNP28666 & A & G & 179 & Syn (Stop) & 0.11 & 0.001795 & 0.09 \\
\hline
\end{tabular}

${ }^{\mathrm{a}} \mathrm{Chr}=$ chromosome; $\mathrm{SNP}=$ single nucleotide polymorphism

${ }^{\mathrm{b}}$ General function classification. A indicates the SNP encodes a regulatory function and B indicates the SNP encodes a transport function.

Table 3. Significant quantitative nucleotide polymorphisms in exons associated (at a false discovery rate threshold of 0.05 ) with deoxynivalenol production in Fusarium graminearum

\begin{tabular}{|c|c|c|c|c|c|c|c|c|}
\hline Chra & SNPa & $\begin{array}{l}\text { Alternative } \\
\text { allele }\end{array}$ & $\begin{array}{l}\text { Reference } \\
\text { allele }\end{array}$ & $\begin{array}{l}\text { Position in } \\
\text { gene }\end{array}$ & Substitution & Frequency & $P$ value & $\begin{array}{c}\text { Explained } \\
\text { genetic variance }\end{array}$ \\
\hline 1 & SNP00102 & $\mathrm{C}$ & A & 831 & Syn (L) & 0.08 & 0.00078 & 0.15 \\
\hline 3 & SNP17537 & $\mathrm{C}$ & $\mathrm{T}$ & 986 & $\mathrm{~V} / \mathrm{A}$ & 0.10 & 0.00113 & 0.15 \\
\hline 2 & SNP12335 & $\mathrm{T}$ & A & 180 & $\mathrm{R} / \mathrm{S}$ & 0.24 & 0.00189 & 0.13 \\
\hline 4 & SNP29587 & $\mathrm{T}$ & $\mathrm{C}$ & 1912 & R/stop & 0.09 & 0.00153 & 0.13 \\
\hline 4 & SNP27625 & $\mathrm{T}$ & $\mathrm{G}$ & 1345 & E/stop & 0.14 & 0.00196 & 0.13 \\
\hline 2 & SNP12939 & G & $\mathrm{C}$ & 331 & $\mathrm{Q} / \mathrm{E}$ & 0.10 & 0.00059 & 0.12 \\
\hline 2 & SNP12986 & A & $\mathrm{C}$ & 995 & $\mathrm{~A} / \mathrm{E}$ & 0.07 & 0.00198 & 0.12 \\
\hline 3 & SNP17581 & $\mathrm{C}$ & $\mathrm{T}$ & 328 & $\mathrm{Y} / \mathrm{H}$ & 0.09 & 0.0013 & 0.12 \\
\hline 4 & SNP23914 & G & $\mathrm{C}$ & 248 & S/stop & 0.11 & 0.00208 & 0.12 \\
\hline 1 & SNP00298 & $\mathrm{T}$ & $\mathrm{C}$ & 360 & Syn $(F)$ & 0.14 & 0.0015 & 0.11 \\
\hline 1 & SNP05884 & G & $\mathrm{C}$ & 1696 & $\mathrm{~L} / \mathrm{V}$ & 0.13 & 0.00183 & 0.11 \\
\hline 2 & SNP12929 & $\mathrm{T}$ & $\mathrm{C}$ & 430 & $\mathrm{P} / \mathrm{S}$ & 0.18 & 0.00123 & 0.11 \\
\hline 3 & SNP17742 & $\mathrm{G}$ & A & 163 & $\mathrm{I} / \mathrm{V}$ & 0.13 & 0.00133 & 0.11 \\
\hline 3 & SNP20351 & $\mathrm{C}$ & $\mathrm{T}$ & 190 & Stop/Q & 0.10 & 0.00148 & 0.11 \\
\hline 1 & SNP06980 & A & G & 1268 & G/D & 0.08 & 0.00164 & 0.10 \\
\hline 1 & SNP00280 & $\mathrm{C}$ & A & 1421 & $\mathrm{E} / \mathrm{A}$ & 0.09 & 0.00161 & 0.09 \\
\hline 1 & SNP03801 & G & A & 1788 & Syn $(\mathrm{K})$ & 0.12 & 0.00213 & 0.08 \\
\hline
\end{tabular}

\footnotetext{
${ }^{\mathrm{a}} \mathrm{Chr}=$ chromosome; SNP = single nucleotide polymorphism.

${ }^{\mathrm{b}}$ General function classification. A indicates the SNP encodes a regulatory function, B indicates the SNP encodes a transport function, and C indicates the SNP encodes a signaling function.
} 
of the variance was in FGSG_12573 (Fig. 4), predicted to encode the enzyme 3-hydroxybutyryl-CoA dehydratase $(h b d)$, a key component of the butyrate production pathway that is involved in spore formation and germination in Clostridium difficile (Pettit et al. 2014).

Production of type-B trichothecene in Trichothecium roseum is based on the trichothecene core structure EPT $(12,13-$ epoxytrichothec-9-ene), in which EPT undergoes hydroxylation at $\mathrm{C}-4(-\mathrm{OH})$ followed by esterification with a butyryl group $(-\mathrm{OR})$, which is then followed by a second hydroxylation at C-8 (-OH) (McCormick et al. 2011). A new trichothecene called NX-2 was recently characterized, using liquid chromatography-tandem mass spectrometry in $F$. graminearum. The nuclear magnetic resonance measurements identified NX-2

Table 2. (continued from previous page)

\begin{tabular}{|c|c|c|c|c|}
\hline $\mathbf{S N P}^{\mathbf{a}}$ & Gene code & Molecular function & Classification $^{\mathbf{b}}$ & Biological processes \\
\hline SNP24237 & FGSG_06583 & ATP binding & A & \\
\hline SNP00852 & FGSG_00355 & RAS GTPas activator & A & RAS GTPase \\
\hline SNP01623 & FGSG_00586 & Metal ion binding & A & Regulation of transcription/DNA-dependent \\
\hline SNP05838 & FGSG_01935 & Transferase activity & $\mathrm{B}$ & GPI anchor biosynthetic process \\
\hline SNP12505 & FGSG_08910 & CAS/CSE protein family & A & Programmed cell death \\
\hline SNP14236 & FGSG_12420 & Hypothetical protein & & \\
\hline SNP25273 & FGSG_13008 & $\begin{array}{l}\text { Metal ion binding/Transcription } \\
\text { factor activity }\end{array}$ & A & Regulation of transcription (Pig1p) \\
\hline SNP07026 & FGSG_12140 & Metallophos 2 & $\mathrm{~B}$ & \\
\hline SNP16744 & FGSG_12204 & Sugar transporter & $\mathrm{B}$ & \\
\hline SNP17675 & FGSG_12685 & NOT2,3,5 domain & A & \\
\hline SNP14402 & FGSG_03620 & Hypothetical protein & & \\
\hline SNP23376 & FGSG_11179 & Hydrolase activity & A & $\begin{array}{l}\text { Secondary metabolism/fumarylacetoacetate } \\
\text { hydrolase }\end{array}$ \\
\hline SNP04014 & FGSG_01339 & DNA binding/TATA binding & A & Regulation of transcription \\
\hline SNP23037 & FGSG_13878 & AMP binding & A & \\
\hline SNP20829 & FGSG_06075 & Microtubule binding/Metal ion binding & A & Protein transport \\
\hline SNP18861 & FGSG_05475 & Protein binding & A & Regulation of transcription \\
\hline SNP29421 & FGSG_09541 & Hydrolase & A & \\
\hline SNP06634 & FGSG_02259 & ATP adenylyltransferase II & A & Phosphate metabolism \\
\hline SNP06829 & FGSG_02352 & Acyltransferase activity & $\mathrm{B}$ & CIT2-citrate (si)-synthase/peroxisomal \\
\hline SNP04746 & FGSG_01563 & Hypothetical protein & & \\
\hline SNP11506 & FGSG_08608 & $\begin{array}{l}\text { PAS2/His Kinase A/Response } \\
\text { Regulator/GAF }\end{array}$ & A & \\
\hline SNP10101 & FGSG_08010 & Zn-cluster & A & Regulatory virulence \\
\hline SNP14633 & FGSG_03751 & L-amino acid transport & $\mathrm{B}$ & $\begin{array}{l}\text { Amino acid derivatives transport/major } \\
\text { facilitator }\end{array}$ \\
\hline SNP07224 & FGSG_02531 & Transcriptional control (zinc binding) & A & Response to stress \\
\hline SNP29198 & FGSG_09604 & Uncharacterized & & \\
\hline SNP28666 & FGSG_13639 & Hypothetical protein & & \\
\hline
\end{tabular}

Table 3. (continued from previous page)

\begin{tabular}{|c|c|c|c|c|}
\hline SNPa & Gene code & Molecular function & Classification $^{b}$ & Biological processes \\
\hline SNP00102 & FGSG_00055 & Hypothetical protein & & \\
\hline SNP17537 & FGSG_05068 & Fungal $\mathrm{Zn}(2)-\mathrm{Cys}(6) / \mathrm{ATP}$ binding & $\mathrm{B}$ & \\
\hline SNP12335 & FGSG_08847 & & & DNA damage response \\
\hline SNP29587 & FGSG_09499 & GTPase activator & A & $\begin{array}{l}\text { Small GTPase mediated signal } \\
\text { transduction }\end{array}$ \\
\hline SNP27625 & FGSG_07578 & ATP binding/transferase activity & $\mathrm{B}$ & Phosphate metabolism \\
\hline SNP12939 & FGSG_12573 & 3-hydroxybutyryl-CoA dehydratase & A & Kinase activity \\
\hline SNP12986 & FGSG_02851 & Fumarylacetoacetase activity & A & \\
\hline SNP17581 & FGSG_12679 & Hypothetical protein & & \\
\hline SNP23914 & FGSG_12927 & Fungal specific transcription factor & $\mathrm{B}$ & \\
\hline SNP00298 & FGSG_11696 & ATP binding & A & DNA repair \\
\hline SNP05884 & FGSG_01943 & PAS & $\mathrm{A} / \mathrm{C}$ & PAS-kinase/UV response \\
\hline SNP12929 & FGSG_02824 & Major facilitator & $\mathrm{B}$ & \\
\hline SNP17742 & FGSG_05119 & Transport facilities & $\mathrm{B}$ & Drug/toxin transport \\
\hline SNP20351 & FGSG_05945 & Hypothetical protein & & \\
\hline SNP06980 & FGSG_02441 & Hydrolase activity & A & \\
\hline SNP00280 & FGSG_00162 & Hypothetical protein & A & \\
\hline SNP03801 & FGSG_01273 & Enzymatic activity regulation & A & Cell growth Morphogenesis \\
\hline
\end{tabular}


as $3 \alpha$-acetoxy-7 $\alpha, 15$-diydroxy-12,13-epoxytrichothec-9-ene, which lacks the keto group at C-8 compared with 3-acetyl (3-)ADON (Varga et al. 2015). In other words, it appears that NX-2 is based on the EPT core structure and requires butyryl for its synthesis. The protein encoded by FGSG_12573 is essential for butyryl synthesis. It is not clear if detection of a significant QTN in FGSG_12573 is connected with the emergence of a new trichothecene variant, because our serological assay could not differentiate between DON and NX-2. But this finding indicates a need to investigate the range of trichothecene variants existing in Europe.

FGSG_01943, explaining 11\% of the genetic variance for DON production, encodes a PAS kinase domain that is orthologous to a gene that coordinates glycogen synthesis in $S$. cerevisiae
(Grose et al. 2007). FGSG_01943 is differentially regulated during crown rot infection in $F$. graminearum compared with ear infection at 2 days postinoculation (Stephens et al. 2008). PAS domains sense redox changes in the electron transport system or the overall redox status and can sense environmental factors affecting cell membranes and cell metabolism (Taylor and Zhulin 1999). In Bordetella species that cause respiratory infection, a PAS protein was linked to a pathogenicity and toxin activator (BvgS protein) and was involved in signaling environmental changes (Taylor and Zhulin 1999). We hypothesize that FGSG_01943 may play a similar role in $F$. graminearum.

A QTN explaining $13 \%$ of the genetic variance was found in gene FGSG_09499, which encodes a GTPase activator

Table 4. Significant quantitative nucleotide polymorphisms in exons associated (at a false discovery rate threshold of 0.05 ) with propiconazole sensitivity in Fusarium graminearum

\begin{tabular}{|c|c|c|c|c|c|c|c|c|}
\hline Chr $^{\mathbf{a}}$ & SNPa & $\begin{array}{c}\text { Alternative } \\
\text { allele }\end{array}$ & $\begin{array}{c}\text { Reference } \\
\text { allele }\end{array}$ & $\begin{array}{l}\text { Position } \\
\text { in gene }\end{array}$ & Substitution & Frequency & $P$ value & $\begin{array}{c}\text { Explained genetic } \\
\text { variance }\end{array}$ \\
\hline 2 & SNP10673 & $\mathrm{T}$ & $\mathrm{C}$ & 62 & Q/Stop & 0.37 & 0.00000094 & 0.17 \\
\hline 3 & SNP19173 & $\mathrm{G}$ & $\mathrm{A}$ & 1221 & Syn (A) & 0.42 & 0.00000086 & 0.17 \\
\hline 4 & SNP23541 & $\mathrm{T}$ & A & 1194 & Syn (T) & 0.44 & 0.00000109 & 0.17 \\
\hline 2 & SNP11749 & A & G & 1898 & $\mathrm{G} / \mathrm{E}$ & 0.25 & 0.00006201 & 0.15 \\
\hline 1 & SNP00477 & G & A & 294 & Syn (E) & 0.25 & 0.0000072 & 0.14 \\
\hline 1 & SNP07664 & A & G & 1008 & Syn (A) & 0.39 & 0.00005009 & 0.14 \\
\hline 1 & SNP06988 & $\mathrm{C}$ & $\mathrm{T}$ & 1104 & Syn (A) & 0.41 & 0.00005273 & 0.14 \\
\hline 1 & SNP04570 & $\mathrm{T}$ & $\mathrm{C}$ & 2908 & $\mathrm{~S} / \mathrm{P}$ & 0.43 & 0.00009546 & 0.14 \\
\hline 4 & SNP26897 & G & $\mathrm{C}$ & 2374 & $\mathrm{G} / \mathrm{R}$ & 0.40 & 0.00002545 & 0.14 \\
\hline 4 & SNP22192 & A & $\mathrm{G}$ & 973 & $\mathrm{~A} / \mathrm{T}$ & 0.08 & 0.00003252 & 0.14 \\
\hline 1 & SNP05545 & $\mathrm{T}$ & $\mathrm{C}$ & 1224 & Stop /Q & 0.47 & 0.00017352 & 0.13 \\
\hline 2 & SNP12056 & $\mathrm{C}$ & $\mathrm{T}$ & 588 & Syn $(\mathrm{N})$ & 0.10 & 0.00226645 & 0.13 \\
\hline 3 & SNP20860 & $\mathrm{T}$ & $\mathrm{C}$ & 2193 & Syn (N) & 0.33 & 0.00009644 & 0.13 \\
\hline 3 & SNP17939 & G & A & 1398 & Syn (Q) & 0.45 & 0.00023358 & 0.13 \\
\hline 4 & SNP28168 & G & A & 249 & Syn (T) & 0.43 & 0.00013855 & 0.13 \\
\hline 4 & SNP24191 & $\mathrm{T}$ & $\mathrm{C}$ & 156 & Syn (F) & 0.41 & 0.00028908 & 0.13 \\
\hline 1 & SNP06462 & $\mathrm{G}$ & A & 2024 & $\mathrm{~K} / \mathrm{R}$ & 0.27 & 0.00120976 & 0.12 \\
\hline 2 & SNP15380 & G & $\mathrm{T}$ & 5905 & $\mathrm{C} / \mathrm{G}$ & 0.10 & 0.0001165 & 0.12 \\
\hline 2 & SNP12563 & $\mathrm{G}$ & $\mathrm{A}$ & 117 & Syn (A) & 0.45 & 0.00060587 & 0.12 \\
\hline 3 & SNP20078 & $\mathrm{A}$ & $\mathrm{C}$ & 2687 & $\mathrm{P} / \mathrm{L}$ & 0.37 & 0.00025337 & 0.12 \\
\hline 3 & SNP21787 & $\mathrm{T}$ & $\mathrm{C}$ & 446 & $\mathrm{P} / \mathrm{L}$ & 0.15 & 0.00092432 & 0.12 \\
\hline 1 & SNP00250 & G & $\mathrm{C}$ & 3343 & $\mathrm{~L} / \mathrm{V}$ & 0.11 & 0.0000995 & 0.11 \\
\hline 1 & SNP086283 & $\mathrm{C}$ & A & 609 & $\mathrm{E} / \mathrm{D}$ & 0.14 & 0.00109387 & 0.11 \\
\hline 1 & SNP07127 & $\mathrm{G}$ & $\mathrm{C}$ & 613 & P/A & 0.18 & 0.00208512 & 0.11 \\
\hline 2 & SNP09492 & $\mathrm{C}$ & $\mathrm{T}$ & 1587 & Syn (I) & 0.11 & 0.00095633 & 0.11 \\
\hline 2 & SNP12939 & $\mathrm{C}$ & A & 726 & $\mathrm{R} / \mathrm{S}$ & 0.14 & 0.0014147 & 0.11 \\
\hline 2 & SNP13914 & $\mathrm{T}$ & $\mathrm{C}$ & 1110 & Syn (D) & 0.26 & 0.00193982 & 0.11 \\
\hline 3 & SNP17476 & $\mathrm{C}$ & $\mathrm{T}$ & 1498 & Syn (L) & 0.08 & 0.00142706 & 0.11 \\
\hline 3 & SNP21737 & G & A & 1333 & $\mathrm{~S} / \mathrm{G}$ & 0.45 & 0.00224438 & 0.11 \\
\hline 4 & SNP21994 & A & $\mathrm{T}$ & 873 & $\operatorname{Syn}(A)$ & 0.20 & 0.00078041 & 0.11 \\
\hline 4 & SNP25548 & A & G & 158 & $\mathrm{D} / \mathrm{S}$ & 0.41 & 0.00079858 & 0.11 \\
\hline 4 & SNP24970 & $\mathrm{C}$ & A & 690 & Syn (V) & 0.11 & 0.00204759 & 0.11 \\
\hline 1 & SNP04911 & A & $\mathrm{C}$ & 2699 & $\mathrm{~A} / \mathrm{E}$ & 0.15 & 0.00050843 & 0.10 \\
\hline 1 & SNP06206 & $\mathrm{C}$ & $\mathrm{G}$ & 1848 & $\mathrm{Q} / \mathrm{H}$ & 0.08 & 0.00121682 & 0.10 \\
\hline 2 & SNP10076 & G & $\mathrm{C}$ & 252 & $\mathrm{D} / \mathrm{E}$ & 0.14 & 0.00070167 & 0.10 \\
\hline 2 & SNP13745 & A & $\mathrm{G}$ & 1033 & $\mathrm{~A} / \mathrm{T}$ & 0.20 & 0.00081782 & 0.10 \\
\hline 2 & SNP09750 & $\mathrm{G}$ & $\mathrm{C}$ & 937 & $\mathrm{~L} / \mathrm{V}$ & 0.10 & 0.00092185 & 0.10 \\
\hline 3 & SNP18638 & $\mathrm{C}$ & $\mathrm{A}$ & 2425 & Syn (R) & 0.25 & 0.00215404 & 0.10 \\
\hline 3 & SNP21657 & $\mathrm{T}$ & A & 970 & $\mathrm{R} / \mathrm{W}$ & 0.11 & 0.00221462 & 0.10 \\
\hline 3 & SNP15606 & $\mathrm{C}$ & $\mathrm{T}$ & 291 & Syn $(\mathrm{H})$ & 0.08 & 0.00222413 & 0.10 \\
\hline 4 & SNP24933 & $\mathrm{T}$ & $\mathrm{A}$ & 754 & $\mathrm{~T} / \mathrm{S}$ & 0.12 & 0.00068842 & 0.10 \\
\hline 1 & SNP01638 & $\mathrm{C}$ & $\mathrm{G}$ & 2467 & $\mathrm{~V} / \mathrm{L}$ & 0.09 & 0.00072028 & 0.09 \\
\hline 1 & SNP03180 & $\mathrm{A}$ & $\mathrm{G}$ & 2064 & Syn (T) & 0.24 & 0.00143591 & 0.09 \\
\hline 1 & SNP00797 & A & $\mathrm{C}$ & 324 & Syn (T) & 0.26 & 0.00149572 & 0.09 \\
\hline 1 & SNP07698 & $\mathrm{G}$ & $\mathrm{A}$ & 3327 & Syn (R) & 0.08 & 0.00197893 & 0.09 \\
\hline 3 & SNP19316 & $\mathrm{T}$ & $\mathrm{A}$ & 644 & $\mathrm{E} / \mathrm{V}$ & 0.19 & 0.00072051 & 0.09 \\
\hline 3 & SNP21836 & $\mathrm{A}$ & $\mathrm{G}$ & 178 & $\mathrm{~A} / \mathrm{T}$ & 0.12 & 0.00075381 & 0.09 \\
\hline 4 & SNP28306 & G & $\mathrm{C}$ & 553 & $\mathrm{P} / \mathrm{A}$ & 0.26 & 0.00093699 & 0.09 \\
\hline 4 & SNP25636 & $\mathrm{C}$ & G & 678 & $\mathrm{~L} / \mathrm{F}$ & 0.10 & 0.00223448 & 0.09 \\
\hline 4 & SNP27287 & G & $\mathrm{C}$ & 649 & H/D & 0.11 & 0.00228217 & 0.09 \\
\hline 1 & SNP02016 & G & $\mathrm{C}$ & 2809 & H/D & 0.18 & 0.0014407 & 0.08 \\
\hline \multicolumn{9}{|c|}{ (continued on next page) } \\
\hline
\end{tabular}

${ }^{\text {a }} \mathrm{Chr}=$ chromosome; $\mathrm{SNP}=$ single nucleotide polymorphism.

${ }^{\mathrm{b}}$ General function classification. A indicates the SNP encodes a regulatory function and B indicates the SNP encodes a transport function. 
domain. The biological function of those genes is not clear, but other genes encoding GTPase were functionally characterized in $F$. graminearum and found to negatively ( FgRacl) or positively $(\mathrm{FgRho4})$ regulate DON production (Zhang et al. 2013).

QTNs were also found in genes encoding a MFS (FGSG_02824, FGSG_05119) and ATP binding domains (FGSG_05068, FGSG_11696, FGSG_07578). A deletion mutant of the Tri12 gene that encodes an MFS in the reference $\mathrm{PH}-1$ strain of $F$. graminearum produced less trichothecene than the wild type (Menke et al. 2012). Earlier studies associated ATP-binding proteins and MFS proteins with secondary metabolites (i.e., mycotoxin production) and resistance to fungicides (Coleman and Mylonakis 2009).

\section{QTNs associated with propiconazole sensitivity.}

Becher et al. (2011) used microarrays to identify 30 genes that were significantly up- or down-regulated upon exposure to azole fungicides in the reference $\mathrm{PH}-1$ strain. Our analyses included 220 wild-type strains sampled from 13 field populations across Germany and coupled natural genetic variation with robust in vitro phenotypic data (Talas and McDonald 2015a) to identify candidate genes for azole sensitivity. None of the genes identified in our GWAS were identified by Becher et al. (2011), likely due to differences in experimental approaches.

The QTN in FGSG_08585 passed the Bonferroni significance threshold and explained $17 \%$ of the genetic variance in azole sensitivity (Fig. 4). This gene encodes an acyl-CoA thioesterase, a protein that plays a role in converting activated

Table 4. (continued from previous page)

\begin{tabular}{|c|c|c|c|c|}
\hline SNPa $^{\mathbf{a}}$ & Gene code & Molecular function & Classification $^{b}$ & Biological process \\
\hline SNP10673 & FGSG_08585 & Acyl-CoA thioesterase & A & Fatty acid metabolism \\
\hline SNP19173 & FGSG_06044 & Translation initiation factor activity & A & Transcriptional control (TAF12) \\
\hline SNP23541 & FGSG_06938 & Metal ion binding/ATP binding & A & Regulation of transcription/RNA splicing \\
\hline SNP11749 & FGSG_08930 & $\mathrm{Zn}(2)-\mathrm{C} 6 /$ Fungal-type DNA binding & A & \\
\hline SNP00477 & FGSG_00259 & Hydrolase activity & A & \\
\hline SNP07664 & FGSG_09919 & Plus-3 (DNA binding) & A & Regulation transcription \\
\hline SNP06988 & FGSG_02619 & Transport & $\mathrm{B}$ & Metal binding \\
\hline SNP04570 & FGSG_01612 & ATP binding & $\mathrm{B}$ & Vesicular transport \\
\hline SNP26897 & FGSG_09611 & $\mathrm{ABC}$ transporter & $\mathrm{B}$ & \\
\hline SNP22192 & FGSG_12935 & Zinc ion binding & A & \\
\hline SNP05545 & FGSG_12042 & C-compound and carbohydrate metabolism & $\mathrm{B}$ & Cofactor transport \\
\hline SNP12056 & FGSG_02836 & Hydrolase activity acting on glycosyl bonds & A & Metabolic process \\
\hline SNP20860 & FGSG_10835 & Hypothetical protein & & \\
\hline SNP17939 & FGSG_05617 & Hypothetical protein & & \\
\hline SNP28168 & FGSG_09088 & C-compound and carbohydrate metabolism & A & Short-chain dehydrogenase \\
\hline SNP24191 & FGSG_13062 & Hypothetical protein & & \\
\hline SNP06462 & FGSG_02390 & Hydrolase activity & A & Metabolic process \\
\hline SNP15380 & FGSG_04588 & Polyketide synthase (PKS29) & $\mathrm{A} / \mathrm{B}$ & Transferase activity, sexual reproduction \\
\hline SNP12563 & FGSG_03100 & Major facilitator & $\mathrm{B}$ & \\
\hline SNP20078 & FGSG_06330 & Damaged DNA binding & A & DNA repair \\
\hline SNP21787 & FGSG_11333 & Hypothetical protein & & \\
\hline SNP00250 & FGSG_11696 & ATP binding/DNA binding & A & $\begin{array}{l}\text { Translation control RNA processing } \\
\text { heavy metal binding }\end{array}$ \\
\hline SNP086283 & FGSG_10282 & Transferase activity & $\mathrm{B}$ & Polysaccharide metabolism \\
\hline SNP07127 & FGSG_02666 & Hydrolase activity Carboxylesterase activity & A & Acetylxylan esterase \\
\hline SNP09492 & FGSG_13455 & DNA binding & A & Transcriptional control \\
\hline SNP12939 & FGSG_03330 & Major facilitator & $\mathrm{B}$ & Multidrug resistance \\
\hline SNP13914 & FGSG_03957 & Cellular import/export transport & $\mathrm{B}$ & \\
\hline SNP17476 & FGSG_05481 & Hypothetical protein & & \\
\hline SNP21737 & FGSG_11301 & Hypothetical protein & & \\
\hline SNP21994 & FGSG_06453 & Major facilitator & $\mathrm{B}$ & C-compound transporter/drug resistance \\
\hline SNP25548 & FGSG_07581 & Hypothetical protein & & \\
\hline SNP24970 & FGSG_13135 & Hypothetical protein & & \\
\hline SNP04911 & FGSG_01735 & $\mathrm{Zn}(2)-\mathrm{C} 6$ fungal-type DNA binding & A & \\
\hline SNP06206 & FGSG_02251 & Metal ion binding/Kinase activity & A & Regulation of transcription \\
\hline SNP10076 & FGSG_08397 & Regulation transcription & A & DNA binding \\
\hline SNP13745 & FGSG_03830 & Sugar transporter/Zn(2)-C6 & $\mathrm{B}$ & \\
\hline SNP09750 & FGSG_08264 & Hypothetical protein & & \\
\hline SNP18638 & FGSG_05884 & ATP binding & A & TATA binding \\
\hline SNP21657 & FGSG_11242 & Iron transporter & $\mathrm{B}$ & \\
\hline SNP15606 & FGSG_04732 & Oxidoreductase activity & A & FAD binding \\
\hline SNP24933 & FGSG_13129 & Cohesin loading factor & A & \\
\hline SNP01638 & FGSG_00627 & Transcription repression & A & \\
\hline SNP03180 & FGSG_01166 & Transport activity & $\mathrm{B}$ & \\
\hline SNP00797 & FGSG_00367 & Hydrolase activity & A & \\
\hline SNP07698 & FGSG_09939 & Hypothetical protein & & \\
\hline SNP19316 & FGSG_06090 & AMP binding & A & Fatty acid metabolic process \\
\hline SNP21836 & FGSG_11383 & Ankyrin repeat & A & Kinetochore complex maturation \\
\hline SNP28306 & FGSG_08995 & Hypothetical protein & & \\
\hline SNP25636 & FGSG_13174 & Heterokaryon incompatibility & A & \\
\hline SNP27287 & FGSG_09509 & ATP binding & A & Hydrolase activity \\
\hline SNP02016 & FGSG_00740 & Hydrolase activity & A & Disease virulence and defense \\
\hline
\end{tabular}


fatty acids into their corresponding nonesterified fatty acids. Hunt and Alexson (2002) proposed that acyl-CoA thioesterase regulates lipid metabolism by maintaining an appropriate balance of acyl-CoA, coenzyme A, and free fatty acids. Sterols and fatty acids, particularly phospholipids, are important components of fungal cell membranes (Pohl et al. 2011). By inhibiting ergosterol synthesis, azole fungicides are likely to cause significant reprogramming of lipid metabolism in fungal cells. Higher amounts of unsaturated lipids were detected, using chromatographic and mass spectrometric techniques, when Taphrina deformans was grown on a medium amended with propiconazole (Weete et al. 1985). The azole fluconazole has more activity against Cryptococcus neoformans when the intracellular fatty acid synthase is inhibited, suggesting that a block in fatty acid synthesis would positively affect an azole's impact on ergosterol synthesis and membrane formation (Chayakulkeeree et al. 2007). Our finding of a QTN in FGSG_08585 indicates that acyl-CoA thioesterase may play an important role in azole sensitivity, possibly through reprogramming the regulation of lipid metabolism in fungal cells exposed to azoles.

Two other QTNs that passed the Bonferroni threshold, each explaining $17 \%$ of the genetic variance, were found in FGSG_06044 and FGSG_06938. FGSG_06044 was predicted to encode the transcription factor TAF12 (= TFIID) and FGSG_06938 encodes a hypothetical protein with different predicted molecular functions (metal ion binding, ATP binding, or RNA splicing), according to the MIPS database. These two genes represent completely novel contributors to antifungal resistance and provide good candidates for future studies on the mechanism of fungicide resistance in field populations of pathogenic fungi.

A DNA mismatch repair function was predicted for the FGSG_06330 protein that explained $12 \%$ of the variation in azole sensitivity (Fig. 4; Table 4). Deletion of genes responsible for DNA mismatch repair in Candida albicans led to an increase in susceptibility to fluconazole (Legrand et al. 2007). Studies of three antifungal agents (amphotericin, miconazole, and ciclopirox) in C. albicans and $S$. cerevisiae showed that DNA damage was significantly associated with antifungal activity and cell death and illustrated that blocking DNA repair could diminish fungicidal activity (Belenky et al. 2013). Our finding suggests a similar association in F. graminearum.

According to the Pfam database (Finn et al. 2014), FGSG_04588 (carrying a QTN explaining 12\% of the genetic variance) encodes the polyketide synthase PKS29 (Sieber et al. 2014). PKS29, together with a terpenoid synthetase and two methyltransferases, is significantly overexpressed $96 \mathrm{~h}$ after infecting wheat and remains highly expressed for 35 days after infection (Sieber et al. 2014). Gaffoor et al. (2005) reported diverse expression patterns in $F$. graminearum for $P K S$ genes during plant colonization, sexual development, and mycelial growth, using gene knockouts. Unfortunately, $P K S 29$ was not included in the study of Gaffoor et al. (2005), so its function in F. graminearum remains unclear. Our findings suggest a possible relationship between polyketide synthases and azole sensitivity in F. graminearum.

Our GWAS analysis detected four QTNs explaining 11, 12, 11 , and $14 \%$ of the genetic variance, respectively, in the genes FGSG_03330, FGSG_03100, FGSG_06453, and FGSG_09611. The first three genes encode proteins in the MFS. FGSG_09611 encodes an ATP-binding cassette (ABC) transporter. The FGSG_ 03330 protein showed a high homology $(83 \%)$ with a multidrug resistance protein in Staphylococcus aureus that acts as an efflux pump (Floyd et al. 2010). The FGSG_03100 and FGSG_06453 proteins showed high homology to MFS proteins characterized in Colletotrichum species. Overexpression of plasma membrane efflux pumps such as MFS and ABC transporters is known to be an important mechanism of azole resistance in many fungi, including
Aspergillus nidulans, Botrytis cinerea, and Zymoseptoria tritici (Cannon et al. 2009). Our findings indicate that these proteins are also important contributors to azole resistance in natural populations of $F$. graminearum.

Our findings illustrate how GWAS has great potential to enable a forward-genetics, bioinformatics-based identification and prioritization of candidate genes affecting important traits in plant-pathogenic fungi. To the best of our knowledge, only three previous GWAS studies were conducted in fungi, including one in Neurospora spp. (Palma-Guerrero et al. 2013), one in S. cerevisiae (Muller et al. 2011), and one in the basidiomycete plant pathogen Heterobasidion annosum (Dalman et al. 2013). Those studies used a relatively small number of isolates (ranging from 23 to 88) and measured traits under highly controlled laboratory conditions. Ours is the first GWAS study to consider important agronomic traits measured under field conditions in an ascomycete plant pathogen.

We identified 26 genes significantly associated with aggressiveness, 17 genes associated with DON production, and 51 genes associated with propiconazole sensitivity in wild-type strains phenotyped under natural field conditions. These candidate genes were distributed randomly across the genome and explained from 8 to $24 \%$ of the genetic variance associated with these traits. Many of the candidate genes do not encode a known function or were not associated with these traits in earlier studies. Targeted functional studies of these genes will be needed to reveal the molecular mechanisms underlying their associations with the respective trait.

\section{MATERIALS AND METHODS}

\section{Phenotyping aggressiveness, mycotoxin production,} and propiconazole sensitivity.

Detailed methods for measuring aggressiveness and mycotoxin production were presented in an earlier publication (Talas et al. 2012a). Briefly, 119 isolates sampled from 13 field populations in Germany (Talas et al. 2011) were phenotyped under field conditions for aggressiveness and mycotoxin production at two different locations over two years (i.e., in four different environments total). Each single-spore isolate came from a different infected wheat spike. Seventy-one isolates were phenotyped (Talas et al. 2012b) in 2009 and 2010 at the experimental field station of the University of Hohenheim $(\mathrm{HOH})$ (longitude $9^{\circ} 12^{\prime} 58^{\prime \prime}$, latitude $48^{\circ} 42^{\prime} 50^{\prime \prime}$, altitude $400 \mathrm{~m}$ ) and at Oberer Lindenhof (OLI) (longitude $9^{\circ} 18^{\prime} 12^{\prime \prime}$, latitude $48^{\circ} 28^{\prime} 26^{\prime \prime}$, altitude $700 \mathrm{~m}$ ). Forty-eight isolates were phenotyped in 2011 and 2012 in $\mathrm{HOH}$ and OLI. The experiments in 2009 and 2010 followed a split-plot design, while the experiments in 2011 and 2012 followed an incomplete block- $\alpha$ design. The weather conditions (temperature and precipitation) differed between $\mathrm{HOH}$ and OLI (Table 5). The experimental plots were arranged in a chessboard pattern of wheat and triticale plots, with each plot measuring $1 \mathrm{~m}$ long and $0.42 \mathrm{~m}$ wide and containing approximately 200 wheat heads per square meter. For all experiments, each isolate was applied to an individual plot planted to the moderately resistant wheat cultivar Taifun (KWS Lochow GMBH, Bergen, Germany) surrounded by four plots of triticale forming a tall border that limited spore movement among experimental plots. For each isolate, a 100-ml spore suspension at a concentration of $2 \times 10^{5}$ spores per milliliter was applied to the heads, using a sprayer with three bars of pressure at growth stage 65 of the Zadoks scale (Zadoks et al. 1974). We phenotyped each isolate in four different environments, and within each environment, the same isolate was phenotyped in two different replicates. Aggressiveness was scored as a single value, including both the number of infected heads per plot and the number of infected spikelets per head, on 
a scale ranging from 0 (no infection) to 100 (each spikelet on each head infected). All wheat heads in each plot were hand-harvested, threshed, and ground to produce flour. Flour (50 g) from each plot was homogenized and extracted to quantify the amount of DON, using a commercial immunoassay kit (Ridascreen Fast Don; R-Biopharm GmbH, Darmstadt, Germany). Because the detection limit of this enzyme-linked immunosorbent assay (ELISA) was $0.23 \mathrm{mg} \mathrm{kg}^{-1}$, smaller values were rounded to zero, resulting in a nonnormal distribution of values. Hence, we followed the suggestion of da Silva et al. (2011) to analyze the DON concentrations following a Box-Cox transformation (using SAS v.9.3) to minimize skewness or kurtosis in the data.

As a consequence of the different experimental designs and different operators involved in data collection, data were analyzed by nesting each group of isolates into its related experimental condition (i.e., $\operatorname{Exp} 1=2009 / 2010$ and $\operatorname{Exp} 2=2011 / 2012$ ). We used the following linear mixed model for the first step of data analysis:

$$
y_{i j k n}=\mu+I s o_{i j}+E n v_{k j}+(I s o \times E n v)_{i k j}+R_{e p} p_{n k}+e_{i j k n},
$$

where $y_{i j k n}$ is the phenotype observation, $\mu$ is the intercept term, $I s o_{i}$ is the genetic effect of the $i$ th isolate in experiment $j$ (i.e., 2009/2010 and 2011/2012), Env $v_{j k}$ is the effect of environment $k$ in the $j$ th experiment, Iso $\times E n v$ is the interaction effect between the $i$ th isolate in the $k$ th environment in the $j$ th experiment, $\operatorname{Rep}_{n k}$ is the effect of the $n$th replicate in the $k$ th environment and $e_{i j k n}$ is the residual error. The variance components were calculated using restricted maximum likelihood. Analyses of phenotypic data were run using SAS v.9.3 software.

The 220 isolates described earlier (Talas and McDonald 2015a), including the 119 isolates phenotyped for aggressiveness and DON production in this analysis, were tested for sensitivity to eight serial dilutions of propiconazole in vitro (i.e., 180, 120, 100, $50,25,10,5$, and $0.5 \mathrm{mg}$ per milliliter) using three technical and two biological repeats of a microtiter plate assay as described earlier (Talas and McDonald 2015a). Briefly, each well contained $10^{5}$ macroconidia, ampicillin and kanamycin at $100 \mathrm{mg}$ per milliliter, and different concentrations of propiconazole in synthetic nutrient broth $\left(0.2 \% \mathrm{KH}_{2} \mathrm{PO}_{4}, 0.2 \% \mathrm{KNO}_{3}, 0.1 \% \mathrm{MgSO}_{4}+7 \mathrm{H}_{2} \mathrm{O}\right.$, $0.1 \% \mathrm{KCl}, 0.2 \%$ sucrose, wt/vol) in a total volume of $200 \mu \mathrm{l}$. After incubating the plates for 7 days in the dark at $24^{\circ} \mathrm{C}$, the optical density (OD) of each well was measured, using an ELISA plate reader, at a wavelength of $405 \mathrm{~nm}$.

The effective fungicide concentration that inhibits isolate growth by $50 \%\left(\mathrm{EC}_{50}\right)$ was calculated for each isolate based on the OD values and according to the following nonlinear model:

$$
y=\min +(\max -\min ) /\left(1+10^{\wedge}\left(\log E C_{50}-x\right) \times \text { Hillslope }\right),
$$

where $y$ was the OD value (in this experiment $y=50$ for $\mathrm{EC}_{50}$ ), min was the minimum OD value corresponding to the highest concentration, max was the maximum OD value corresponding to the lowest concentration; $x$ was the propiconazole concentration related to the $y$ response, and Hillslope was the steepness of the dose/response curve. $\mathrm{EC}_{50}$ values of all isolates were analyzed using SAS v.9.3 software, as described earlier (Talas and McDonald 2015a).

Proc Univariate was used to verify the normal distribution of the residuals for all measured traits. Heritability $\left(H^{2}\right)$ for aggressiveness, DON production, and propiconazole sensitivity was estimated as the ratio of genotypic variance to the total phenotypic variance (Fehr 1987). These analyses were performed using SAS v.9.3.

\section{Genotyping based on RADseq.}

Genomic data for $220 \mathrm{~F}$. graminearum isolates was obtained using RADseq (Hohenlohe et al. 2010). Briefly, genomic DNA of each isolate was digested with PstI and two different barcodes were ligated onto the resulting sticky ends. Afterward, different libraries were established for each barcode, were pooled into two lanes, and were sequenced using 100-bp paired-end reads on an Illumina HiSeq2000. The quality of the resulting data were checked using FASTX toolkit v.0.13. All sequences with average raw-data quality lower than 20 were discarded, using Trimmomatic v. 0.32 (Bolger et al. 2014) applied on a sliding window of $5 \mathrm{bp}$. Only sequences longer than $30 \mathrm{bp}$ were retained. All alignments were performed using Bowtie2 v.2.2.0 (Langmead and Salzberg 2012). Samtools 0.1.19 (Li et al. 2009) was used to sort and index the sequences based on their barcodes. We used the published sequence of the reference isolate $F$. graminearum $\mathrm{PH}-1$ (FG3, Broad Institute) to call the SNPs using the Genome Analysis Tool Kit v.3.1.1 (McKenna et al. 2010). Filtering conditions were set as minimum sequence quality $=600$ and minimum sequence depth $=20$ (Talas and McDonald 2015b). SNPs that did not meet these criteria were discarded using VCFtools v.3.5 (Danecek et al. 2011). These filtering steps resulted in 36,283 high quality SNPs distributed over the four chromosomes. To avoid biases in association analyses due to imbalanced allele frequencies (Massman et al. 2010), the minimum allele frequency was set to $7 \%$ and the maximum allele frequency was set to $92 \%$. After further filtering to allow a maximum of $3.9 \%$ missing data for any SNP locus, we identified 30,797 SNPs to use for the aggressiveness and DON production traits and 28,440 SNPs for the propiconazole sensitivity trait. The SNP dataset is available online at the European Nucleotide Archive (ENA) under the study name: ena-STUDY ETH-09-10-2015-11:15:47:949-18 and the accession number PRJEB11357. Use of these data requires citation.

\section{GWAS analyses.}

A population genetic analysis based on a subset of 1,129 RADseq SNPs distributed evenly across the genome showed an

\begin{tabular}{|c|c|c|c|c|c|c|c|}
\hline \multirow[b]{2}{*}{ Station $^{\mathbf{a}}$} & \multirow[b]{2}{*}{ Year } & \multicolumn{3}{|c|}{ Average over the year } & \multicolumn{3}{|c|}{ Average during the growing season ${ }^{b}$} \\
\hline & & Day temp. $\left({ }^{\circ} \mathrm{C}\right)$ & Night temp. $\left({ }^{\circ} \mathrm{C}\right)$ & Precipitation (mm) & Day temp. $\left({ }^{\circ} \mathrm{C}\right)$ & Night temp. $\left({ }^{\circ} \mathrm{C}\right)$ & Precipitation (mm) \\
\hline OLI & 2009 & 11.5 & 4.3 & 990.3 & 20.6 & 11.5 & 104.4 \\
\hline OLI & 2010 & 9.8 & 3.1 & 989.7 & 20.1 & 11.6 & 126.9 \\
\hline OLI & 2011 & 12.8 & 4.7 & 804.4 & 20.0 & 10.8 & 104.1 \\
\hline OLI & 2012 & 11.2 & 3.8 & 915.8 & 20.9 & 11.5 & 136.4 \\
\hline $\mathrm{HOH}$ & 2009 & 14.4 & 5.7 & 767.4 & 23.7 & 13.3 & 108.7 \\
\hline $\mathrm{HOH}$ & 2010 & 12.9 & 5 & 621.7 & 23.6 & 13.7 & 94.4 \\
\hline $\mathrm{HOH}$ & 2011 & 15.5 & 6 & 571.2 & 23.1 & 13.1 & 98.5 \\
\hline $\mathrm{HOH}$ & 2012 & 14.7 & 5.8 & 684.2 & 24.2 & 13.7 & 67.3 \\
\hline
\end{tabular}

Table 5. Climatic conditions in field sites where aggressiveness and deoxynivalenol production were measured

a OLI = Oberer Lindenhof, $\mathrm{HOH}=$ Hohenheim.

b The period from first of June to end of August, including the heading and ripening stages in wheat associated with Fusarium head blight infection and covering the latent and infectious periods for the pathogen. 
absence of population structure among the isolates included in the GWAS analyses, even though these isolates were sampled from wheat fields across Germany (Talas and McDonald 2015b). This suggests that a single meta-population of $F$. graminearum is distributed across the sampled geographical region in Germany. The same dataset exhibited a rapid decay in LD within a physical distance of $1 \mathrm{~kb}$ across all chromosomes, indicating a high resolution would be possible for association studies (Talas and McDonald 2015b). Consequently, no correction for population structure or LD was performed for this dataset. However, we removed highly linked SNP markers, using the PLINK v. 1.07 package (Purcell et al. 2007), resulting in a final filtered total of 29,949 SNPs analyzed for the aggressiveness and DON production traits and 28,038 SNPs analyzed for the propiconazole resistance trait.

The same mixed linear model was applied for each trait following the model:

$$
y=a+b x+g+e,
$$

where $y$ is the phenotype, $a$ is the mean term, $b$ is the additive effect (fixed effect) of the candidate SNP to be tested for association, $x$ is the allele effect, $g$ is the polygenic effect (random effect), and $e$ is the residual. The analysis was performed using the GCTA v.1.24 package (Yang et al. 2011). The polygenic effects have a distribution with a mean of zero and a covariance matrix $g=2 k \sigma_{a}^{2}$, where $k$ is the kinship (coancestry) matrix with a pairwise estimate, and $\sigma^{2}$ is an unknown genetic variance (Zhang et al. 2010). The significance of marker-phenotype association was tested using the Wald $F$ test with a significance threshold of $P<0.05$. All results were adjusted using the Bonferroni correction and FDR procedure as implemented in the PLINK v. 1.07 software package (Purcell et al. 2007).

The variance explained by a single causal variant depends upon the allelic effect size and its frequency within the tested data. The genetic variance contributed by each SNP was calculated separately through the formula:

$$
R^{2}=2 p(1-p) a^{2},
$$

where $p$ is the frequency of the causal variant and $a$ is the effect size on an arbitrary scale (Robinson et al. 2014). The explained variance algorithm was implemented in the GABIT software package (Lipka et al. 2012). The total genetic effect of each isolate was calculated using the BLUP (best linear unbiased prediction) approach based on the combined effects of the SNPs. These results were then transformed into a BLUP solution for each SNP effect, based on a kinship matrix. $R^{2}$ refers to the genetic portion of the phenotypic variance. Values of $R^{2}$ were calculated for each QTN separately (Sun et al. 2010). The resulting $P$ values were plotted against the expected $P$ values, to show how well the applied model with kinship correction fit the data. The negative logarithmic values of the resulting $P$ values were plotted against the allele frequency of each SNP to approximate the percentage of isolates sharing the same allele at each QTN. The resulting QTNs were plotted against the logarithmic value of their significance in a Manhattan plot. All figures were generated using the $\mathrm{R}$ package (available online).

\section{Assignment of candidate genes and predicting their function.}

The SNP position on the reference genome was combined with the chromosome number to identify genes containing significant QTNs using CLC Genomics Workbench 6 (CLC Inc., Aarhus, Denmark). The sequence of each candidate gene was translated and analyzed for any possible domains using the Pfam 29.0 database (Finn et al. 2014). In addition, each protein sequence was blasted using the National Center for
Biotechnology Information database. In parallel, the molecular and biological function of each candidate gene was investigated using the MIPS database for $F$. graminearum. We focused our attention on genes with well-known functions in $F$. graminearum and genes with clearly identified domains possessing high homology with other pathogenic fungi.

\section{ACKNOWLEDGMENTS}

The authors thank both the Plant Fellows program (FP7 Marie Curie Actions) and ETH Zurich for funding the current study. The agricultural experiment station at University of Hohenheim was used to generate the field data. B. Lieberherr assisted with the field experiment. The genome datasets were generated using the facilities of the Genetic Diversity Center (GDC) at ETH Zurich.

\section{LITERATURE CITED}

Baird, N. A., Etter, P. D., Atwood, T. S., Currey, M. C., Shiver, A. L., Lewis, Z. A., Selker, E. U., Cresko, W. A., and Johnson, E. A. 2008. Rapid SNP discovery and genetic mapping using sequenced RAD markers. PLoS One 3:e3376.

Barrett, R. D. H., and Hoekstra, H. E. 2011. Molecular spandrels: Tests of adaptation at the genetic level. Nat. Rev. Genet. 12:767-780.

Bartels, D. J., Mitchell, D. A., Dong, X., and Deschenes, R. J. 1999. Erf2, a novel gene product that affects the localization and palmitoylation of Ras2 in Saccharomyces cerevisiae. Mol. Cell. Biol. 19:6775-6787.

Becher, R., Miedaner, T., and Wirsel, S. G. R. 2013. Biology, diversity, and management of FHB-causing Fusarium species in small-grain cereals. Page 199-241 in: Agricultural Applications. F. Kempken, ed. Springer, Heidelberg.

Becher, R., Weihmann, F., Deising, H. B., and Wirsel, S. G. 2011. Development of a novel multiplex DNA microarray for Fusarium graminearum and analysis of azole fungicide responses. BMC Genomics 12:52.

Belenky, P., Camacho, D., and Collins, J. J. 2013. Fungicidal drugs induce a common oxidative-damage cellular death pathway. Cell Reports 3:350-358.

Benjamini, Y., and Hochberg, Y. 1995. Controlling the false discovery rate-A practical and powerful approach to multiple testing. J. R. Stat. Soc. 57:289-300

Bluhm, B. H., Zhao, X., Flaherty, J. E., Xu, J. R., and Dunkle, L. D. 2007. RAS2 regulates growth and pathogenesis in Fusarium graminearum. Mol. Plant-Microbe Interact. 20:627-636.

Boenisch, M. J., and Schäfer, W. 2011. Fusarium graminearum forms mycotoxin producing infection structures on wheat. BMC Plant Biol. 11: 110.

Bolger, A. H., Lohse, M., and Usadel, B. 2014. Trimmomatic A flexible trimmer for illumina sequence data. Bioinformatics 30:2114-2120.

Brinkmann, U., Brinkmann, E., Gallo, M., Scherf, U., and Pastan, I. 1996. Role of CAS, a human homologue to the yeast chromosome segregation gene CSE1, in toxin and tumor necrosis factor mediated apoptosis Biochemistry 35:6891-6899.

Brunner, P. C., Stefanato, F. L., and McDonald, B. A. 2008. Evolution of the CYP51 gene in Mycosphaerella graminicola: Evidence for intragenic recombination and selective replacement. Mol. Plant Pathol. 9:305-316.

Burke, M. K., King, E. G., Shahrestani, P., Rose, M. R., and Long, A. D. 2014. Genome-wide association study of extreme longevity in Drosophila melanogaster. Genome Biol. Evol. 6:1-11.

Cannon, R. D., Lamping, E., Holmes, A. R., Niimi, K., Baret, P. V., Keniya, M. V., Tanabe, K., Niimi, M., Goffeau, A., and Monk, B. C. 2009. Effluxmediated antifungal drug resistance. Clin. Microbiol. Rev. 22:291-321.

Chayakulkeeree, M., Rude, T. H., Toffaletti, D. L., and Perfect, J. R. 2007. Fatty acid synthesis is essential for survival of Cryptococcus neoformans and a potential fungicidal target. Antimicrob. Agents Chemother. 51: 3537-3545.

Coleman, J. J., and Mylonakis, E. 2009. Efflux in fungi: La pièce de résistance. PLoS Pathog. 5:e1000486.

Cuomo, C. A., Güldener, U., Xu, J. R., Trail, F., Turgeon, B. G., Di Pietro, A., Walton, J. D., Ma, L. J., Baker, S. E., Rep, M., Adam, G., Antoniw, J., Baldwin, T., Calvo, S., Chang, Y. L., Decaprio, D., Gale, L. R., Gnerre, S., Goswami, R. S., Hammond-Kosack, K., Harris, L. J., Hilburn, K., Kennell, J. C., Kroken, S., Magnuson, J. K., Mannhaupt, G., Mauceli, E., Mewes, H. W., Mitterbauer, R., Muehlbauer, G., Münsterkötter, M., Nelson, D., O'donnell, K., Ouellet, T., Qi, W., Quesneville, H., Roncero, M. I., Seong, K. Y., Tetko, I. V., Urban, M., Waalwijk, C., Ward, T. J., Yao, J., Birren, B. W., and Kistler, H. C. 2007. The Fusarium 
graminearum genome reveals a link between localized polymorphism and pathogen specialization. Science 317:1400-1402.

da Silva, M. V. G. B., Van Tassell, C. P., Sonstegard, T. S., Cobuci, J. A., and Gasbarre, L. C. 2011. Box-Cox transformation and random regression models for fecal egg count data. Front. Genet. 2:112.

Dalman, K., Himmelstrand, K., Olson, A., Lind, M., Brandström-Durling, M., and Stenlid, J. 2013. A genome-wide association study identifies genomic regions for virulence in the non-model organism Heterobasidion annosum s.s. PLoS One 8:e53525.

Danecek, P., Auton, A., Abecasis, G., Albers, C. A., Banks, E., DePristo, M. A., Handsaker, R. E., Lunter, G., Marth, G. T., Sherry, S. T., McVean, G., and Durbin, R.; 1000 Genomes Project Analysis Group. 2011. The variant call format and VCFtools. Bioinformatics 27:2156-2158.

Dubos, T., Pasquali, M., Pogoda, F., Hoffmann, L., and Beyer, M. 2011. Evidence for natural resistance towards trifloxystrobin in Fusarium graminearum. Eur. J. Plant Pathol. 130:239-248.

Fehr, W. R. 1987. Principles of Cultivar Development. Macmillan, New York.

Finn, R. D., Bateman, A., Clements, J., Coggill, P., Eberhardt, R. Y., Eddy, S. R., Heger, A., Hetherington, K., Holm, L., Mistry, J., Sonnhammer, E. L. L., Tate, J., and Punta, M. 2014. Pfam: The protein families database. Nucleic Acids Res. 42:D222-D230.

Floyd, J. L., Smith, K. P., Kumar, S. H., Floyd, J. T., and Varela, M. F. 2010 LmrS is a multidrug efflux pump of the major facilitator superfamily from Staphylococcus aureus. Antimicrob. Agents Chemother. 54:5406-5412.

Frazer, K. A., Murray, S. S., Schork, N. J., and Topol, E. J. 2009. Human genetic variation and its contribution to complex traits. Nat. Rev. Genet. 10:241-251.

Frishman, D., Mokrejs, M., Kosykh, D., Kastenmüller, G., Kolesov, G., Zubrzycki, I., Gruber, C., Geier, B., Kaps, A., Albermann, K., Volz, A., Wagner, C., Fellenberg, M., Heumann, K., and Mewes, H. W. 2003. The PEDANT genome database. Nucleic Acids Res. 31:207-211.

Frohner, I. E., Bourgeois, C., Yatsyk, K., Majer, O., and Kuchler, K. 2009. Candida albicans cell surface superoxide dismutases degrade hostderived reactive oxygen species to escape innate immune surveillance. Mol. Microbiol. 71:240-252.

Gaffoor, I., Brown, D. W., Plattner, R., Proctor, R. H., Qi, W., and Trail, F. 2005. Functional analysis of the polyketide synthase genes in the filamentous fungus Gibberella zeae (anamorph: Fusarium graminearum). Eukaryot. Cell 4:1926-1933.

Grose, J. H., Smith, T. L., Sabic, H., and Rutter, J. 2007. Yeast PAS kinase coordinates glucose partitioning in response to metabolic and cell integrity signaling. EMBO (Eur. Mol. Biol. Organ.) J. 26:4824-4830.

Harashima, T., Anderson, S., Yates, J. R., 3rd, and Heitman, J. 2006. The kelch proteins Gpb1 and Gpb2 inhibit Ras activity via association with the yeast RasGAP neurofibromin homologs Ira1 and Ira2. Mol. Cell 22:819-830

Hochberg, Y. 1988. A sharper Bonferroni procedure for multiple tests of significance. Biometrika 75:800-802.

Hohenlohe, P. A., Bassham, S., Etter, P. D., Stiffler, N., Johnson, E. A., and Cresko, W. A. 2010. Population genomics of parallel adaptation in threespine stickleback using sequenced RAD tags. PLoS Genet. 6:e1000862.

Hou, Z., Xue, C., Peng, Y., Katan, T., Kistler, H. C., and Xu, J.-R. 2002. A mitogen-activated protein kinase gene (MGV1) in Fusarium graminearum is required for female fertility, heterokaryon formation, and plant infection. Mol. Plant-Microbe Interact. 15:1119-1127.

Hunt, M. C., and Alexson, S. E. H. 2002. The role Acyl-CoA thioesterases play in mediating intracellular lipid metabolism. Prog. Lipid Res. 41: 99-130.

Kang, H. M., Zaitlen, N. A., Wade, C. M., Kirby, A., Heckerman, D., Daly, M. J., and Eskin, E. 2008. Efficient control of population structure in model organism association mapping. Genetics 178:1709-1723.

King, R., Urban, M., Hammond-Kosack, M. C. U., Hassani-Pak, K., and Hammond-Kosack, K. E. 2015. The completed genome sequence of the pathogenic ascomycete fungus Fusarium graminearum. BMC Genomics 16:544.

Langmead, B., and Salzberg, S. 2012. Fast gapped-read alignment with Bowtie 2. Nat. Method 9:357-359.

Lawler, K., Hammond-Kosack, K., Brazma, A., and Coulson, R. M. R. 2013. Genomic clustering and co-regulation of transcriptional networks in the pathogenic fungus Fusarium graminearum. BMC Syst. Biol. 7:52.

Lee, N., and Kronstad, J. W. 2002. ras2 Controls morphogenesis, pheromone response, and pathogenicity in the fungal pathogen Ustilago maydis. Eukaryot. Cell 1:954-966.

Legrand, M., Chan, C. L., Jauert, P. A., and Kirkpatrick, D. T. 2007. Role of DNA mismatch repair and double-strand break repair in genome stability and antifungal drug resistance in Candida albicans. Eukaryot. Cell 6:2194-2205

Li, H., Handsaker, B., Wysoker, A., Fennell, T., Ruan, J., Homer, N., Marth, G., Abecasis, G., Durbin, R., and 1000 Genome Project Data Processing Subgroup. 2009. The sequence alignment/map (SAM) format and SAMtools. Bioinformatics 25:2078-2079.

Lipka, A. E., Tian, F., Wang, Q., Peiffer, J., Li, M., Bradbury, P. J., Gore, M. A., Buckler, E. S., and Zhang, Z. 2012. GAPIT: Genome association and prediction integrated tool. Bioinformatics 28:2397-2399.

Massman, J., Cooper, B., Horsley, R., Neate, S., Dill-Macky, R., Chao, S., Dong, Y., Schwarz, P., Muehlbauer, G. J., and Smith, K. P. 2010. Genome-wide association mapping of Fusarium head blight resistance in contemporary barley breeding germplasm. Mol. Breed. 27:439-454

McCarthy, M. I., Abecasis, G. R., Cardon, L. R., Goldstein, D. B., Little, J., Ioannidis, J. P. A., and Hirschhorn, J. N. 2008. Genome-wide association studies for complex traits: Consensus, uncertainty and challenges. Nat. Rev. Genet. 9:356-369.

McCormick, S. P., Harris, L. J., Alexander, N. J., Ouellet, T., Saparno, A., Allard, S., and Desjardins, A. E. 2004. Tri1 in Fusarium graminearum encodes a P450 oxygenase. Appl. Environ. Microbiol. 70:2044-2051.

McCormick, S. P., Stanley, A. M., Stover, N. A., and Alexander, N. J. 2011. Trichothecenes: From simple to complex mycotoxins. Toxins (Basel) 3:802-814

McKenna, A., Hanna, M., Banks, E., Sivachenko, A., Cibulskis, K., Kernytsky, A., Garimella, K., Altshuler, D., Gabriel, S., Daly, M., and DePristo, M. A 2010. The genome analysis toolkit: A MapReduce framework for analyzing next-generation DNA sequencing data. Genome Res. 20:1297-1303.

Meek, I. B., Peplow, A. W., Jr., Ake, C., Jr., Phillips, T. D., and Beremand, M. N. 2003. Tri1 encodes the cytochrome P450 monooxygenase for C-8 hydroxylation during trichothecene biosynthesis in Fusarium sporotrichioides and resides upstream of another new Tri gene. Appl. Environ. Microbiol. 69:1607-1613.

Menke, J., Dong, Y., and Kistler, H. C. 2012. Fusarium graminearum Tri12p influences virulence to wheat and trichothecene accumulation. Mol. Plant-Microbe Interact. 25:1408-1418.

Muller, L. A. H., Lucas, J. E., Georgianna, D. R., and McCusker, J. H. 2011. Genome-wide association analysis of clinical vs. nonclinical origin provides insights into Saccharomyces cerevisiae pathogenesis. Mol. Ecol. 20:4085-4097.

Nelson, R. M., Pettersson, M. E., Li, X., and Carlborg, O. 2013. Variance heterogeneity in Saccharomyces cerevisiae expression data: Transregulation and epistasis. PLoS One 8:e79507.

Palma-Guerrero, J., Hall, C. R., Kowbel, D., Welch, J., Taylor, J. W., Brem, R. B., and Glass, N. L. 2013. Genome wide association identifies nove loci involved in fungal communication. PLoS Genet. 9:e1003669.

Pettit, L. J., Browne, H. P., Yu, L., Smits, W. K., Fagan, R. P., Barquist, L., Martin, M. J., Goulding, D., Duncan, S. H., Flint, H. J., Dougan, G., Choudhary, J. S., and Lawley, T. D. 2014. Functional genomics reveals that Clostridium difficile Spo0A coordinates sporulation, virulence and metabolism. BMC Genomics 15:160.

Pohl, C. H., Kock, J., and Thibane, V. S. 2011. Antifungal free fatty acids: A review. Pages 693-1348 in: Science Against Microbial Pathogens: Communicating Current Research and Technology Advances. A. Mendez-Vilas, ed. Formatex Research Center, Badajoz, Spain.

Purcell, S., Neale, B., Todd-Brown, K., Thomas, L., Ferreira, M. A. R., Bender, D., Maller, J., Sklar, P., de Bakker, P. I. W., Daly, M. J., and Sham, P. C. 2007. PLINK: A tool set for whole-genome association and population-based linkage analyses. Am. J. Hum. Genet. 81:559-575.

Qin, J., Wang, G., Jiang, C., Xu, J.-R., and Wang, C. 2015. Fgk3 glycogen synthase kinase is important for development, pathogenesis, and stress responses in Fusarium graminearum. Sci. Rep. 5:8504.

Rietveld, C. A., Medland, S. E., Derringer, J., Yang, J., Esko, T., Martin, N. W., Westra, H.-J., Shakhbazov, K., Abdellaoui, A., Agrawal, A., Albrecht, E., Alizadeh, B. Z., Amin, N., Barnard, J., Baumeister, S. E., Benke, K. S., Bielak, L. F., Boatman, J. A., Boyle, P. A., Davies, G., de Leeuw, C., Eklund, N., Evans, D. S., Ferhmann, R., Fischer, K., Gieger, C., Gjessing, H. K., Hägg, S., Harris, J. R., Hayward, C., Holzapfel, C. Ibrahim-Verbaas, C. A., Ingelsson, E., Jacobsson, B., Joshi, P. K., Jugessur, A., Kaakinen, M., Kanoni, S., Karjalainen, J., Kolcic, I., Kristiansson, K., Kutalik, Z., Lahti, J., Lee, S. H., Lin, P., Lind, P. A., Liu, Y., Lohman, K., Loitfelder, M., McMahon, G., Vidal, P. M., Meirelles, O., Milani, L., Myhre, R., Nuotio, M. L., Oldmeadow, C. J., Petrovic, K. E., Peyrot, W. J., Polasek, O., Quaye, L., Reinmaa, E., Rice, J. P., Rizzi, T. S., Schmidt, H., Schmidt, R., Smith, A. V., Smith, J. A., Tanaka, T., Terracciano, A., van der Loos, M. J., Vitart, V., Völzke, H., Wellmann, J., Yu, L., Zhao, W., Allik, J., Attia, J. R., Bandinelli, S., Bastardot, F., Beauchamp, J., Bennett, D. A., Berger, K., Bierut, L. J., Boomsma, D. I., Bültmann, U., Campbell, H., Chabris, C. F., Cherkas, 
L., Chung, M. K., Cucca, F., de Andrade, M., De Jager, P. L., De Neve, J. E., Deary, I. J., Dedoussis, G. V., Deloukas, P., Dimitriou, M., Eiríksdóttir, G., Elderson, M. F., Eriksson, J. G., Evans, D. M., Faul, J. D., Ferrucci, L., Garcia, M. E., Grönberg, H., Guðnason, V., Hall, P., Harris, J. M., Harris, T. B., Hastie, N. D., Heath, A. C., Hernandez, D. G., Hoffmann, W., Hofman, A., Holle, R., Holliday, E. G., Hottenga, J. J., Iacono, W. G., Illig, T., Järvelin, M. R., Kähönen, M., Kaprio, J., Kirkpatrick, R. M., Kowgier, M., Latvala, A., Launer, L. J., Lawlor, D. A., Lehtimäki, T., Li, J., Lichtenstein, P., Lichtner, P., Liewald, D. C., Madden, P. A., Magnusson, P. K., Mäkinen, T. E., Masala, M., McGue, M., Metspalu, A., Mielck, A., Miller, M. B., Montgomery, G. W., Mukherjee, S., Nyholt, D. R., Oostra, B. A., Palmer, L. J., Palotie, A., Penninx, B. W., Perola, M., Peyser, P. A., Preisig, M., Räikkönen, K., Raitakari, O. T., Realo, A., Ring, S. M., Ripatti, S., Rivadeneira, F., Rudan, I., Rustichini, A., Salomaa, V., Sarin, A. P., Schlessinger, D., Scott, R. J., Snieder, H., St Pourcain, B., Starr, J. M., Sul, J. H., Surakka, I., Svento, R., Teumer, A., Tiemeier, H., van Rooij, F. J., Van Wagoner, D. R., Vartiainen, E., Viikari, J., Vollenweider, P., Vonk, J. M., Waeber, G., Weir, D. R., Wichmann, H. E., Widen, E., Willemsen, G., Wilson, J. F., Wright, A. F., Conley, D., Davey-Smith, G., Franke, L., Groenen, P. J., Hofman, A., Johannesson, M., Kardia, S. L., Krueger, R. F., Laibson, D., Martin, N. G., Meyer, M. N., Posthuma, D., Thurik, A. R., Timpson, N. J., Uitterlinden, A. G., van Duijn, C. M., Visscher, P. M., Benjamin, D. J., Cesarini, D., and Koellinger, P. D.; LifeLines Cohort Study. 2013. GWAS of 126,559 individuals identifies genetic variants associated with educational attainment. Science 340:1467-1471.

Riley, M. L., Schmidt, T., Artamonova, I. I., Wagner, C., Volz, A., Heumann, K., Mewes, H.-W., and Frishman, D. 2007. PEDANT genome database: 10 years online. Nucleic Acids Res. 35:D354-D357.

Robinson, M. R., Wray, N. R., and Visscher, P. M. 2014. Explaining additional genetic variation in complex traits. Trends Genet. 30:124-132.

Shlezinger, N., Goldfinger, N., and Sharon, A. 2012. Apoptotic-like programed cell death in fungi: The benefits in filamentous species. Front. Oncol. 2:97.

Sieber, C. M. K., Lee, W., Wong, P., Münsterkötter, M., Mewes, H.-W., Schmeitzl, C., Varga, E., Berthiller, F., Adam, G., and Güldener, U. 2014. The Fusarium graminearum genome reveals more secondary metabolite gene clusters and hints of horizontal gene transfer. PLoS One 9:e110311.

Stephens, A. E., Gardiner, D. M., White, R. G., Munn, A. L., and Manners, J. M. 2008. Phases of infection and gene expression of Fusarium graminearum during crown rot disease of wheat. Mol. Plant-Microbe Interact. 21:1571-1581.

Sun, G., Zhu, C., Kramer, M. H., Yang, S.-S., Song, W., Piepho, H. P., and Yu, J. 2010. Variation explained in mixed-model association mapping. Hered. Edinb 105:333-340.

Sun, J., Kale, S. P., Childress, A. M., Pinswasdi, C., and Jazwinski, S. M. 1994. Divergent roles of RAS1 and RAS2 in yeast longevity. J. Biol. Chem. 269:18638-18645

Talas, F., Kalih, R., and Miedaner, T. 2012a. Within-field variation of Fusarium graminearum isolates for aggressiveness and deoxynivalenol production in wheat head blight. Phytopathology 102:128-134.

Talas, F., and McDonald, B. A. 2015a. Significant variation in sensitivity to a DMI fungicide in field populations of Fusarium graminearum. Plant Pathol. 64:664-670.

Talas, F., and McDonald, B. A. 2015b. Genome-wide analysis of Fusarium graminearum field populations reveals hotspots of recombination. BMC Genomics 16:996.
Talas, F., Parzies, H. K., and Miedaner, T. 2011. Diversity in genetic structure and chemotype composition of Fusarium graminearum sensu stricto populations causing wheat head blight in individual fields in Germany. Eur. J. Plant Pathol. 131:39-48.

Talas, F., Würschum, T., Reif, J. C., Parzies, H. K., and Miedaner, T. 2012b. Association of single nucleotide polymorphic sites in candidate genes with aggressiveness and deoxynivalenol production in Fusarium graminearum causing wheat head blight. BMC Genet. 13:14.

Taylor, B. L., and Zhulin, I. B. 1999. PAS domains: Internal sensors of oxygen, redox potential, and light. Microbiol. Mol. Biol. Rev. 63:479-506.

Varga, E., Wiesenberger, G., Hametner, C., Ward, T. J., Dong, Y., Schöfbeck, D., McCormick, S., Broz, K., Stückler, R., Schuhmacher, R., Krska, R., Kistler, H. C., Berthiller, F., and Adam, G. 2015. New tricks of an old enemy: Isolates of Fusarium graminearum produce a type A trichothecene mycotoxin. Environ. Microbiol. 17:2588-2600.

Voigt, C. A., Schäfer, W., and Salomon, S. 2005. A secreted lipase of Fusarium graminearum is a virulence factor required for infection of cereals. Plant J. 42:364-375.

Wagacha, J. M., and Muthomi, J. W. 2007. Fusarium culmorum: Infection process, mechanisms of mycotoxin production and their role in pathogenesis in wheat. Crop Prot. 26:877-885.

Wang, C., Zhang, S., Hou, R., Zhao, Z., Zheng, Q., Xu, Q., Zheng, D., Wang, G., Liu, H., Gao, X., Ma, J. W., Kistler, H. C., Kang, Z., and Xu, J. R. 2011. Functional analysis of the kinome of the wheat scab fungus Fusarium graminearum. PLoS Pathog. 7:e1002460.

Weete, J. D., Sancholle, M., and Montant, C. 1985. Effects of triazoles on fungi. II. Composition of a plasma membrane-enriched fraction of Taphrina deformans. Biochim. Biophys. Acta 812:633-642.

Wong, P., Walter, M., Lee, W., Mannhaupt, G., Münsterkötter, M., Mewes, H.-W., Adam, G., and Güldener, U. 2011. FGDB: Revisiting the genome annotation of the plant pathogen Fusarium graminearum. Nucleic Acids Res. 39:D637-D639.

Xiao, B.-Z., Chen, X., Xiang, C.-B., Tang, N., Zhang, Q.-F., and Xiong, L.-Z. 2009. Evaluation of seven function-known candidate genes for their effects on improving drought resistance of transgenic rice under field conditions. Mol. Plant 2:73-83.

Yang, J., Lee, S. H., Goddard, M. E., and Visscher, P. M. 2011. GCTA: A tool for genome-wide complex trait analysis. Am. J. Hum. Genet. 88:76-82.

Zadoks, J. C., Chang, T. T., and Konzak, C. F. 1974. A decimal code for growth stages of cereals. Weed Res. 14:415-421.

Zhang, C., Wang, Y., Wang, J., Zhai, Z., Zhang, L., Zheng, W., Zheng, W., Yu, W., Zhou, J., Lu, G., Shim, W.-B., and Wang, Z. 2013. Functional characterization of Rho family small GTPases in Fusarium graminearum. Fungal Genet. Biol. 61:90-99.

Zhang, Z., Ersoz, E., Lai, C.-Q., Todhunter, R. J., Tiwari, H. K., Gore, M. A., Bradbury, P. J., Yu, J., Arnett, D. K., Ordovas, J. M., and Buckler, E. S. 2010. Mixed linear model approach adapted for genome-wide association studies. Nat. Genet. 42:355-360.

Zhao, C., Waalwijk, C., de Wit, P. J. G. M., Tang, D., and van der Lee, T. 2013. RNA-Seq analysis reveals new gene models and alternative splicing in the fungal pathogen Fusarium graminearum. BMC Genomics $14: 21$.

\section{AUTHOR-RECOMMENDED INTERNET RESOURCE}

The R Project for Statistical Computing: http://www.R-project.org 